\title{
REPRESENTATION THEOREMS FOR PARABOLIC SYSTEMS
}

\section{J. CHABROWSKI}

(Received 14 May 1981)

Communicated by R. Vyborny

\begin{abstract}
The aim of this article is to review the progress made in the last few years in the representation theory of solutions of parabolic systems in the sense of Petrowskii.

1980 Mathematics subject classification (Amer. Math. Soc.): primary 35 K 30, 35 K 45; secondary 35 C 15.
\end{abstract}

\section{Introduction}

In theory of partial differential equations of parabolic type the notion of the fundamental solution plays a very important role. It is widely known that most quantitive statements concerning the solutions of parabolic differential equations and systems are derived from propeties of the fundamental solution. The construction of the fundamental solution based on the parametrix method has been known for a long time. The fundamental solutions for second-order parabolic equations were constructed by M. Gevrey, F. G. Dressel, W. Pogorzelski and D. G. Aronson. Subsequently the parametrix method was extended to systems of equations parabolic in the sense of I. G. Petrowski by W. Pogorzelski, O. A. Ladyzhenskaja, D. G. Aronson and S. D. Eidel'man.

The principal objective of this exposition is the development of the theory of the representation of solutions, primarily of the Cauchy problem for parabolic systems and equations and the related behaviour of the solutions near the boundary. It is of considerable interest that all these considerations are entirely

C. Copyright Australian Mathematical Society 1982 
based on properties of the fundamental solution and some results of measure theory in $R_{n}$.

The prototype of representation theory is the following result for the heat equation.

Let $u$ be a solution of the heat equation

$$
\Delta_{x} u-u_{t}=0 \text { for }(x, t) \in R_{n} \times(0, T]
$$

such that $\int_{R_{n}}|u(x, t)|^{P} e^{-\beta|x|^{2}} d x \quad(1 \leqslant p \leqslant \infty)$ is bounded for $t \in(0, T]$, then there exists a unique function $f$ such that $f e^{-\beta|x|^{2}} \in L^{P}\left(R_{n}\right)(1<p<\infty)$ and

$$
u(x, t)=\int_{R_{n}}(4 \pi t)^{-n / 2} \exp \left(-\frac{|x-y|^{2}}{4 t}\right) f(y) d y
$$

for $(x, t) \in R_{n} \times(0, T]$. If $p=1$, then

$$
u(x, t)=\int_{R_{n}}(4 \pi t)^{-n / 2} \exp \left(\frac{|x-y|^{2}}{4 t}\right) \mu(d y),
$$

where $\mu$ is a Borel measure in $R_{n}$ such that

$$
\int_{R_{n}} e^{-\beta|x|^{2}}|\mu|(d x)<\infty
$$

In this context non-negative solutions are of special interest since in physical phenomena the solutions of parabolic equations usually represent positive quantities such as temperatures, densities, probabilities etc. Widder (1944) discovered that if $u$ is non-negative solution of the heat equation then $\int_{R_{n}} u(x, t) e^{-\beta|x|^{2}} d x$ is bounded in $(0, T]$, where $\beta$ is a positive constant. Hence in the integral representation formula $\mu$ is a non-negative Borel measure. It is worth mentioning here that the function $(4 \pi t)^{-n / 2} \exp \left(-|x|^{2} / 4 t\right)$ appearing in the representation formula is the fundamental solution of the heat equation. The above integral representation guarantees the existence of the limit of $u$ almost everywhere in $R_{n}$ as $t \rightarrow 0$. This gives an extension of the classical Fatou theorem known for analytic and harmonic functions, and allows the introduction of the notion of parabolic limit which corresponds to the non-tangential limit in the case of harmonic functions.

Section 1 provides the basic material for the study of properties of the fundamental matrix of a parbolic system of any order.

In Section 2 we concentrate on the representation theorems of solutions in Orlicz spaces.

In Section 3 we give the notion of a parabolic limit and prove the Fatou property.

Section 4 is devoted to study parabolic systems of the second order. We prove a necessary and sufficient condition for the fundamental matrix to have nonnegative elements. As a result we obtain that a parabolic system of second order 
having non-negative elements in its fundamental matrix must be weakly coupled. A significant feature of weakly coupled parabolic systems is that the classical maximum principal is applicable. This makes possible the investigation of properties of non-negative solutions in greater detail.

In the preceding considerations there is a lower estimate for the diagonal elements of the fundamental matrix which plays an essential part.

In Section 6 we proceed to apply some results on differentiation of positive measures to investigate the behaviour of non-negative solution near the boundary.

In Sections 7 and 8 we are concerned with the rate of spacial decay of non-negative solutions and the local Fatou property.

Finally, in Section 9 we briefly discuss Widder's Inversion Theorem and its connection with the initial distribution problem. The results of Section 7 are joint with N. A. Watson. Certain results of this paper (in particular of Section 8) are published here for the first time.

The author is indebted to $\mathbf{H}$. B. Thompson for valuable and stimulating comments on the subject.

\section{Fundamental solutions for parabolic systems of the order $2 m$}

This section provides the fundamental material required for our study of the representation of solutions of parabolic systems in the sense of Petrowskii. We state in this section some of the main results about fundamental solutions which will be needed in the subsequent presentation of the subject.

Consider the $N \times N$ system of equations

$$
\frac{\partial u_{j}}{\partial t}=\sum_{j=1}^{N} \sum_{|k|<2 m} A_{k}^{i j}(x, t) D_{x}^{k} u_{j}+g_{i}(x, t) \quad(i=1, \ldots, N),
$$

where $k=\left(k_{1}, \ldots, k_{n}\right),|k|=k_{1}+\cdots+k_{n}, D_{x}^{k}=D_{x_{1}}^{k_{1}} \cdots D_{x_{n}}^{k_{n}}, D_{x_{1}}=\frac{\partial}{\partial x_{1}}$. The functions $g_{i}(x, t)$ and the coefficients $A_{k}^{i j}$ are defined on $R_{n} \times[0, T]$.

Let

$$
\frac{\partial u_{j}}{\partial t}=\sum_{j=1}^{N} \sum_{|k|<2 m} A_{k}^{i j}(x, t) D_{x}^{k} u_{j}
$$

be the corresponding homogeneous system.

Consider the determinant

$$
\operatorname{det}\left[\sum_{|k|=2 m} A_{k}^{r s}(x, t)(i \xi)^{k}-\delta_{r s} \lambda\right]
$$


where $\xi=\left(\xi_{1}, \ldots, \xi_{n}\right)$ is a real vector with norm $|\xi|=\left(\sum_{i-1}^{n} \xi_{i}^{2}\right)^{1 / 2}=1, \delta_{r s}$ is the Kronecker symbol, $i=\sqrt{-1}$, and $(i \xi)^{k}=\left(i \xi_{1}\right)^{k_{1}} \cdots\left(i \xi_{n}\right)^{k_{n}}$. Denote the roots of the determinant (2) by $\lambda_{j}(\xi ; x, t)$.

We say that the system (1) (or (1h)) is uniformly parabolic in $R_{n} \times[0, T]$ (in the sense of Petrowskii) if there exists a constant $\delta>0$ such that

$$
\max _{j} \sup _{|\xi|=1} \operatorname{Re}\left\{\lambda_{j}(\xi ; x, t)\right\}<-\delta
$$

for all $(x, t) \in R_{n} \times[0, T]$.

The Cauchy problem for the system (1) consists in finding a solution for (1) in the strip $R_{n} \times(0, T]$ satisfying the initial conditions

$$
u_{i}(x, t)=\phi_{i}(x) \quad(i=1, \ldots, N),
$$

where the $\phi_{i}(x)$ are given functions defined on $\boldsymbol{R}_{\boldsymbol{n}}$.

By a fundamental solution (or a fundamental matrix) $\Gamma(x, t ; y, \tau)=$ $\left\{\Gamma_{i j}(x, t ; y, \tau)\right\}(i, j=1, \ldots, N)$ we mean an $N \times N$ matrix defined for $(x, t)$, $(y, \tau) \in R_{n} \times[0, T], \tau<t$, which, as a function of $(x, t)\left(x \in R_{n}, \tau<t<T\right)$, satisfies (1h) (that is, each column is a solution of (1h)), and is such that for each continuous and bounded function $f(x)$ on $R_{n}$, we have

$$
\lim _{t \rightarrow \tau^{+}} \int_{R_{n}} \Gamma_{i j}(x, t ; y, \tau) f(y) d y=\delta_{i j} f(x) \quad(i, j=1, \ldots, N)
$$

at every point $x \in R_{n}$.

Throughout we shall make the following assumptions:

$\left(\mathrm{A}_{1}\right)$ The coefficients of $(\mathrm{lh})$ are continuous, bounded functions on $R_{n} \times$ $[0, T]$ and, furthermore, the principal coefficients (with $|k|=2 m$ ) are continuous in $t$, uniformly with respect to $(x, t) \in R_{n} \times[0, T]$.

$\left(A_{2}\right)$ The coefficients of $(\mathrm{lh})$ are Hölder continuous of exponent $\alpha(0<\alpha<1)$ in $x$, uniformly with respect to $(x, t)$ in bounded subsets of $R_{n} \times[0, T]$, and, furthermore, the principal coefficients are Hölder continuous of exponent $\alpha$ $(0<\alpha<1)$ in $t$, uniformly with respect to $(x, t) \in R_{n} \times[0, T]$.

THEOREM 1.1. Assume that (1) is uniformly parabolic in $R_{n} \times[0, T]$ and that $\left(\mathrm{A}_{1}\right),\left(\mathrm{A}_{2}\right)$ holds. Then there exists a fundamental solution $\Gamma(x, t ; y, \tau)$ of $(1 \mathrm{~h})$ satisfying the inequalities

$$
\begin{aligned}
& \left|D_{x}^{k} \Gamma(x, t ; y, \tau)\right| \leqslant C(t-\tau)^{-(n+|k|) / 2 m} \exp \left[-\chi\left(\frac{|x-y|^{2 m}}{t-\tau}\right)^{1 /(2 m-1)}\right], \\
& \left|D_{t} \Gamma(x, t ; y, \tau)\right| \leqslant C(t-\tau)^{-(n+2 m) / 2 m} \exp \left[-\chi\left(\frac{|x-y|^{2 m}}{t-\tau}\right)^{1 /(2 m-1)}\right]
\end{aligned}
$$

for all $(x, t),(y, \tau) \in R_{n} \times[0, T],-\tau<t,|k|<2 m$, where $C$ and $\chi$ are positive constants. 
With the aid of the fundamental solution we can solve the Cauchy problem (1), (3). By a solution of the problem (1), (3) in the strip $R_{n} \times(0, T]$ we mean functions $\left\{u_{i}(x, t)\right\} \quad(i=1, \ldots, N)$ which satisfy (1) for $(x, t) \in R_{n} \times$ $(0, T]\left(\partial u_{i} / \partial t\right.$ and $\left.D_{x}^{k} u_{i}, 0<|k| \leqslant 2 m, i=1, \ldots, N\right)$ are assumed to exist and be continuous on $R_{n} \mid(0, T]$, and which are continuous on $R_{n} \times[0, T]$ and satisfy (3) for $x \in R_{n}$.

THEOREM 1.2. Assume that the system (1) is uniformly parabolic in $R_{n} \times[0, T]$ and that $\left(\mathrm{A}_{1}\right),\left(\mathrm{A}_{2}\right)$, hold in $R_{n} \times[0, T]$. Let $\left\{g_{i}(x, t)\right\}(i=1, \ldots, N)$ be continuous functions in $R_{n} \times[0, T]$, Hölder continuous in $t$ uniformly in bounded subsets of $R_{n} \times[0, T]$, and let $\left\{\phi_{i}(x)\right\}(i=1, \ldots, N)$ be continuous functions on $R_{n}$. Moreover, suppose that,

$$
\begin{gathered}
\int_{0}^{T} \int_{R_{n}}\left|g_{i}(x, t)\right|^{p} \exp \left(-a|x|^{2 m /(2 m-1)}\right) d x d t<\infty, \\
\int_{R_{n}}\left|\phi_{i}(x)\right|^{p} \exp \left(-a|x|^{2 m /(2 m-1)}\right) d x<\infty \quad(i=1, \ldots, N)
\end{gathered}
$$

for some constants $1 \leqslant p<\infty, 0 \leqslant a<\infty$.

Then there exists a solution of the Cauchy problem (1), (3) in a strip $R_{n} \times$ $\left(0, T_{1}\right], T_{1} \leqslant T$, given by the formula

$$
\begin{aligned}
u_{i}(x, t)= & \int_{R_{n}} \sum_{j=1}^{N} \Gamma_{i j}(x, t ; y, 0) \phi_{j}(y) d y \\
& -\int_{0}^{t} d \tau \int_{R_{n}} \sum_{j=1}^{N} \Gamma_{i j}(x, t ; y, \tau) g_{j}(y, \tau) d y \quad(i=1, \ldots, N) .
\end{aligned}
$$

To define the adjoint system of $(\mathrm{lh})$ we shall need the following assumption:

$\left(\mathrm{A}_{3}\right)$ The derivatives $D_{x}^{h} A_{k}^{i j}(x, t)(0 \leqslant|h| \leqslant|k|)$ are continuous bounded functions in $R_{n} \times[0, T]$, and Hölder continuous of exponent $\alpha(0<\alpha<1)$ in $x$, uniformly with respect to $(x, t)$ in bounded subsets of $R_{n} \times[0, T]$.

The adjoint system of $(1 \mathrm{~h})$ is given by

$$
\frac{\partial v_{i}}{\partial t}=-\sum_{j=1}^{N} \sum_{|k|<2 m}(-1)^{|k|} D_{x}^{k}\left[A_{k}^{j i}(x, t) v_{j}\right] .
$$

A fundamental solution (or fundamental matrix) of (9) is an $N \times N$ matrix $\Gamma^{*}(x, t ; y, \tau)=\left\{\Gamma_{i j}^{*}(x, t ; y, \tau)\right\}$ defined for $(x, t),(y, \tau) \in R_{n} \times[0, T], t<\tau$, which as a function of $(x, t)\left(x \in R_{n}, 0 \leqslant t<\tau\right)$ satisfies (9), and is such that for each function $f(x)$, that is continuous and bounded on $R_{n}$, we have

$$
\lim _{t \rightarrow \tau-} \int_{R_{n}} \Gamma_{i j}^{*}(x, t ; y, \tau) f(y) d y=\delta_{i j} f(x) \quad(i, j=1, \ldots, N) .
$$


THEOREM 13. Let the system (1h) be uniformly parabolic in $R_{n} \times[0, T]$, and let the assumptions $\left(\mathrm{A}_{1}\right),\left(\mathrm{A}_{2}\right)$ and $\left(\mathrm{A}_{3}\right)$ hold. Then there exists a fundamental solution $\Gamma^{*}$ of the system (9) such that

$$
\Gamma^{*}(y, \tau ; x, t)=\Gamma(x, t ; y, \tau)^{T}
$$

for all $(x, t),(y, \tau) \in R_{n} \times[0, T], \tau<t$, where $\Gamma^{T}$ denotes the transpose of $\Gamma$.

THEOREM 1.4. Let the system (1h) be uniformly parabolic in $R_{n} \times[0, T]$ and let the assumptions $\left(\mathrm{A}_{1}\right),\left(\mathrm{A}_{2}\right)$ and $\left(\mathrm{A}_{3}\right)$ hold. Then there exists at most one solution of the Cauchy problem (1), (3) satisfying for some $a>0$.

$$
\int_{0}^{T} \int_{R_{n}}\left|u_{i}(x, t)\right| \exp \left(-a|x|^{2 m /(2 m-1)}\right) d x d t<\infty \quad(i=1, \ldots, N) .
$$

It follows from the estimates (5) that if $f_{j}(x) \exp \left(-\beta|x|^{2 m /(2 m-1)}\right) \in L^{p}\left(R_{n}\right)$ $(j=1, \ldots, N), 1 \leqslant p<\infty$, for some positive constant $\beta$, then

$$
u_{i}(x, t)=\int_{R_{n}} \sum_{j=1}^{N} \Gamma_{i j}(x, t ; y, 0) f_{j}(y) d y
$$

is a solution of (1h) in a strip $R_{n} \times\left(0, T_{1}\right], T_{1}<T$. Moreover one can prove that for some constant $B$, say, we have

$$
\left\|u_{i}(\cdot, t) \exp \left(-\beta|\cdot|^{2 m /(2 m-1)}\right)\right\|_{L^{p}\left(R_{n}\right)} \leqslant B \sum_{j=1}^{N}\left\|f_{j}(\cdot) \exp \left(-\beta|\cdot|^{2 m /(2 m-1)}\right)\right\|_{L^{p}\left(R_{n}\right)}
$$

(if $p=\infty$ we use the supremum norm rather than $L^{p}$-norm) and

$$
\left\|u_{i}(\cdot, t)-f_{i}(\cdot) \exp \left(-\beta|\cdot|^{2 m /(2 m-1)}\right)\right\|_{L^{p}\left(R_{n}\right)} \rightarrow 0 \quad(i=1, \ldots, N)
$$

as $t \rightarrow 0$, if $1 \leqslant p<\infty$.

Finally we note that under the assumptions $\left(A_{1}\right),\left(A_{2}\right)$ and $\left(A_{3}\right)$ the fundamental matrix satisfies the Kolmogorov identity

$$
\Gamma_{i j}(x, t ; y, \tau)=\int_{R_{n}} \sum_{k=1}^{N} \Gamma_{i k}(x, t ; z, \delta) \Gamma_{k j}(z, \delta ; y, \tau) d z \quad(i, j=1, \ldots, N)
$$

for all $x, y \in R_{n}, 0 \leqslant \tau<\delta<t \leqslant T$.

The proofs of Theorems 1.1-1.4 can be found in the monographs of Eidel'man (1969) and Friedman (1964); see these books for their historical references.

\section{Representation theorems in Orlicz spaces}

In this section we establish some preliminary result about the uniqueness and representation of solution of the Cauchy problem in Orlicz spaces. Extensive studies of Orlicz spaces can be found in the monographs Kransnosel'skii and Rutickii (1961) and Kufner, John, Fučik (1977). 
A function $\phi$ defined on $[0, \infty)$ is a Young function if

$$
\Phi(t)=\int_{0}^{t} \phi(s) d s \text { for } t>0,
$$

where the function $\phi$ defined on $[0, \infty)$ has the following properties:

(i) $\phi(0)=0, \phi(t)>0$ for $t>0$,

(ii) $\phi$ is right-hand continuous on $[0, \infty)$,

(iii) $\phi$ is non-decreasing on $[0, \infty)$,

(iv) $\lim _{s \rightarrow \infty} \phi(s)=\infty$.

Let $\Phi$ be a Young function generated by the function $\phi$, put

$$
\psi=\sup _{\phi(s)<t} s
$$

and

$$
\Psi(t)=\int_{0}^{t} \psi(s) d s
$$

The function $\Psi$ is called the complementary function to $\Phi$ and it is also a Young function.

Put

$$
\rho(u ; \Phi)=\int_{R_{n}} \Phi(|u(x)|) d x .
$$

Let $\Phi$ and $\Psi$ be a pair of complementary Young functions and let $u$ be a measurable function on $R_{n}$, the number

$$
\|u\|_{\Phi}=\sup _{v} \int_{R_{n}}|u(x) v(x)| d x,
$$

where the supremum is taken over all measurable function $v$ on $R_{n}$ such that $\rho(v ; \Psi) \leqslant 1$, is called the Orlicz norm. The set of all measurable functions $u$ on $R_{n}$ such that $\|u\|_{\Phi}<\infty$ is denoted by $L_{\Phi}\left(R_{n}\right)$ and called the Orlicz space.

Analogously we can define the weighted Orlicz space $L_{\Phi}^{\alpha, \gamma}\left(R_{n}\right)$ with respect to the finite measure $\exp \left(-\alpha|x|^{\gamma}\right) d x$, where $\alpha$ and $\gamma$ are positive constants.

Denote by $\tilde{L}_{\Phi}^{\alpha, \gamma}\left(R_{n}\right)$ the set of all measurable functions $u$ on $R_{n}$ such that

$$
\int_{R_{n}} \Phi(|u(x)|) \exp \left(-\alpha|x|^{\gamma}\right) d x<\infty .
$$

It is easy to see that $\int_{R} \Phi\left(\exp \left(-\alpha|x|^{\gamma}\right)\right) d x<\infty$ for all $\alpha>0$ and $\gamma>0$. Consequently if $u \in \tilde{L}_{\Phi}^{\alpha, \gamma}\left(R_{n}\right)$ then $u(x) \exp \left(-\beta|x|^{\gamma}\right) \in L^{1}\left(R_{n}\right)$ for all $\beta>\alpha$. 
Indeed by the generalized Hölder inequality and the convexity of $\Phi$ we obtain

$$
\begin{aligned}
& \int_{R_{n}}|u(x)| \exp \left(-\beta|x|^{\gamma}\right) d x \\
& \quad<\int_{R_{n}} \Phi\left(\exp \left(-\alpha|x|^{\gamma}\right)|u(x)|\right) d x \int_{R_{n}} \Psi\left(\exp \left(-(\beta-\alpha)|x|^{\gamma}\right)\right) d x \\
& \quad<\int_{R_{n}} \Phi(|u(x)|) \exp \left(-\alpha|x|^{\gamma}\right) d x \int_{R_{n}} \Psi\left(\exp \left(-(\beta-\alpha)|x|^{\gamma}\right)\right) d x .
\end{aligned}
$$

From now on we shall adopt the following

$$
\tilde{L}_{\Phi}^{\alpha, 2 m /(2 m-1)}\left(R_{n}\right)=\tilde{L}_{\Phi}^{\alpha}\left(R_{n}\right), \quad L_{\Phi}^{\alpha, 2 m /(2 m-1)}\left(R_{n}\right)=L_{\Phi}^{\alpha}\left(R_{n}\right) .
$$

THEOREM 2.1. Let the system (1h) be uniformly parabolic in $R_{n} \times[0, T]$ and let the assumptions $\left(\mathrm{A}_{1}\right)$ and $\left(\mathrm{A}_{2}\right)$ hold. Let $f_{j} \in \tilde{L}_{\Phi}^{\alpha}\left(R_{n}\right)(j=1, \ldots, N)$, then

$$
u_{i}(x, t)=\int_{R_{n}} \sum_{j=1}^{N} \Gamma_{i j}(x, t ; y, 0) f_{j}(y) d y \quad(i=1, \ldots, N)
$$

is a solution of the system (1h) in $R_{n} \times\left(0, T_{1}\right]$, where $T_{1}<T$. Moreover, there exists a positive constant $C$ such that

$$
\begin{aligned}
& \left\|u_{j}(\cdot, t) \exp \left(-2^{2 m /(2 m-1)} \alpha|\cdot|^{2 m /(2 m-1)}\right)\right\|_{\Phi} \\
& \quad<C \sum_{j=1}^{N} \int_{R_{n}} \Phi\left(\exp \left(-\alpha|x|^{2 m /(2 m-1)}\left|f_{j}(x)\right|\right)\right) d x \quad(i=1, \ldots, N)
\end{aligned}
$$

for $t \in\left(0, T_{1}\right]$.

Proof. The first part of the theorem follows from the inequality (14) and Theorem 1.2.

To establish (16) let $v$ be an arbitrary function such that $\rho(v ; \Psi)<1$. By (5) we have

$$
\begin{aligned}
\int_{R_{n}}\left|u_{i}(x, t)\right| & \exp \left(-2^{2 m /(2 m-1)} \alpha|x|^{2 m(2 m-1)}\right)|v(x)| d x \\
< & \int_{R_{n}}\left[\int_{R_{n} j=1} \sum_{i j}^{N}\left|\Gamma_{i j}(x, t ; y, 0) f_{j}(y)\right| d y\right] \\
& \times \exp \left(-2^{2 m /(2 m-1)} \alpha|x|^{2 m /(2 m-1)}\right)|v(x)| d x \\
< & C \int_{R_{n}}\left[\int_{R_{n} j=1}^{N} \exp \left(-\chi|z|^{2 m /(2 m-1)}\right)\left|f_{j}\left(x+t^{2 m /(2 m-1)} z\right)\right| d z\right] \\
& \times \exp \left(-2^{2 m /(2 m-1)} \alpha|x|^{2 m /(2 m-1)}\right)|v(x)| d x .
\end{aligned}
$$


On the other hand

$$
\begin{aligned}
& \exp \left(-2^{2 m /(2 m-1)} \alpha|x|^{2 m / 2 m-1)}\right) \\
& \quad \leqslant \exp \left(-\alpha\left|x+t^{2 m /(2 m-1)} z\right|^{2 m /(2 m-1)}+2^{2 m /(2 m-1)} \alpha|z|^{2 m /(2 m-1)} t^{1 /(2 m-1)}\right) .
\end{aligned}
$$

Combining this with (17) and the generalized Hölder inequality the result follows provided $0<t \leqslant T_{1}<(\chi / \alpha)^{2 m-1} 2^{-2 m}$.

Similarly using the estimates (5) one can prove

LEMMA 2.1. Assume that (1h) is uniformly parabolic in $R_{n} \times[0, T]$ and that $\left(\mathrm{A}_{1}\right),\left(\mathrm{A}_{2}\right)$ hold. Let $\left\{u_{i}(x, t)\right\}(i=1, \ldots, N)$ be a solution of $(\mathrm{lh})$ in $R_{n} \times(0, T]$ such that

$$
\int_{R_{n}} \Phi\left(\left|u^{i}(x, t)\right|\right) \exp \left(-\alpha|x|^{2 m /(2 m-1)}\right) d x<M
$$

for $0<t \leqslant T$, where $M$ is a positive constant, then there exists a positive constant $\delta$ such that for all $0<\tau<T$

$$
u_{i}(x, t)=\int_{R_{n}} \sum_{j=1}^{N} \Gamma_{i j}(x, t ; y, \tau) u_{j}(y, \tau) d y \quad(i=1, \ldots, N)
$$

in $R_{n} \times(\tau, \min (T, \tau+\delta)$, where $\delta$ is independent of $\tau$.

By $E_{\Phi}^{\alpha}\left(R_{n}\right)$ we denote the closure in $L_{\Phi}^{\alpha}\left(R_{n}\right)$ of the set of all bounded measurable functions on $R_{n}$.

In the next theorem we shall need the following property of the space $L_{\Phi}^{\alpha}\left(R_{n}\right)$ : every bounded sequence in $L_{\Phi}^{\alpha}$ contains an $E_{\Psi}^{\alpha}$-weakly convergent subsequence ( $\Psi$ is the complementary function to $\Phi$ ).

Now we are in a position to establish the converse theorem to Theorem 2.1.

TheOREM 2.2. Assume that the system (1h) is uniformly parabolic in $R_{n} \times[0, T]$ and that $\left(\mathrm{A}_{1}\right),\left(\mathrm{A}_{2}\right)$ and $\left(\mathrm{A}_{3}\right)$ hold. Let $\left\{u_{i}(x, t)\right\}(i=1, \ldots, N)$ be a solution of (1h) in $R_{n} \times(0, T]$ such that

$$
\int_{R_{n}} \Phi\left(\left|u_{i}(x, t)\right|\right) \exp \left(-\alpha|x|^{2 m /(2 m-1)}\right) d x<M \quad(i=1, \ldots, N)
$$

for all $0<t \leqslant T$, where $M$ and $\alpha$ are positive constants. Then there exist unique functions $f_{j} \in \tilde{L}_{\Phi}^{\alpha}\left(R_{n}\right)$ such that

$$
u_{i}(x, t)=\int_{R_{n} j=1} \sum_{j=1}^{N} \Gamma_{i j}(x, t ; y, 0) f_{j}(y) d y \quad(i=1, \ldots, N)
$$

for all $(x, t) \in R_{n} \times(0, T]$. 
Proof. It follows from (18) that $\left\{u_{i}(\cdot, t), 0<t<T\right\}(i=1, \ldots, N)$ are bounded subsets of $L_{\Phi}^{\alpha}\left(R_{n}\right)$. Hence there exist a sequence $\left\{t_{\nu}\right\}$ converging to 0 and functions $f_{i} \in L_{\Phi}^{\alpha}\left(R_{n}\right)(i=1, \ldots, N)$ such that

$$
\begin{aligned}
& \lim _{\nu \rightarrow \infty} \int_{R_{n}} u_{i}\left(x, t_{v}\right) \exp \left(-\alpha|x|^{2 m /(2 m-1)}\right) v(x) d x \\
& \quad=\int_{R_{n}} f_{i}(x) \exp \left(-\alpha|x|^{2 m /(2 m-1)}\right) v(x) d x \quad(i=1, \ldots, N)
\end{aligned}
$$

for every $v \in E_{\Psi}^{\alpha}\left(R_{n}\right)$. By Lemma 2.1, Theorem 1.4 and the inequality (14) there exists $\delta>0$ such that

$$
u_{i}(x, t)=\int_{R_{n}} \sum_{j=1}^{N} \Gamma_{i j}\left(x, t ; y, t_{v}\right) u_{j}\left(y, t_{\nu}\right) d y \quad(i=1, \ldots, N)
$$

for $0<t_{y}<t \leqslant \delta, x \in R_{n}$. Consequently

$$
\begin{aligned}
u_{i}(x, t) & -\int_{R_{n}} \sum_{j=1}^{N} \Gamma_{i j}(x, t ; y, 0) f_{j}(y) d y \\
= & {\left[\int_{R_{n}} \sum_{j=1}^{N} \Gamma_{i j}\left(x, t ; y, t_{v}\right) u_{j}\left(y, t_{v}\right) d y-\int_{R_{n} j=1} \sum_{i j}^{N} \Gamma_{i j}(x, t ; y, 0) u_{j}\left(y, t_{v}\right) d y\right] } \\
& +\left[\int_{R_{n}} \sum_{j=1}^{N} \Gamma_{i j}(x, t ; y, 0) u_{j}\left(y, t_{v}\right) d y-\int_{R_{n} j=1} \sum_{i j}^{N} \Gamma_{i j}(x, t ; y, 0) f_{j}(y) d y\right] \\
= & J_{1}+J_{2} .
\end{aligned}
$$

Since $\Gamma_{i j}(x, t ; \cdot, 0) \exp \left(\alpha|\cdot|^{2 m /(2 m-1)}\right) \in E_{\Psi}^{\alpha}\left(R_{n}\right)$ (for sufficiently small $t$ ), $\lim _{v \rightarrow \infty} J_{2}=0$. To prove that $\lim _{v \rightarrow \infty} J_{1}=0$, let us observe by (18) we have $\left|J_{1}\right|<M \sum_{j=1}^{N} \int_{R} \Psi\left(\left|\Gamma_{i j}\left(x, t ; y, t_{v}\right)-\Gamma_{i j}(x, t ; y, 0)\right| \exp \left(\alpha|y|^{2 m /(2 m-1)}\right)\right) d y$.

It is easy to show, using (5), that the last integrand converges uniformly to 0 on $R_{n}$, provided $\delta$ is sufficiently small. To prove that formula (19) holds on $R_{n} \times(0, T]$ suppose that $(x, t) \in R_{n} \times(\delta, 2 \delta]$. Thus by Lemma 2.1 and Kolmogorov's identity

$$
\begin{aligned}
u_{i}(x, t) & =\int_{R_{n}} \sum_{j=1}^{N} \Gamma_{i j}(x, t ; y, \delta) u_{j}(y, \delta) d y \\
& =\int_{R_{n}}\left[\int_{R_{n}} \sum_{j=1}^{N} \Gamma_{i j}(x, t ; y, \delta) \sum_{k=1}^{N} \Gamma_{j k}(y, \delta ; z, 0) f_{k}(z) d z\right] d y \\
& =\int_{R_{n}} \sum_{k=1}^{N} \Gamma_{i k}(x, t ; z, 0) f_{k}(z) d z \quad(i=1, \ldots, N) .
\end{aligned}
$$


We can now proceed step by step to prove that formula (19) holds in $R_{n} \times$ $(0, T]$. The uniqueness of $f_{j}$ is a consequence of the Fatou property which will be stated in the next section.

As a particular case we obtain

Corollary 2.1. Suppose that the hypotheses $\left(\mathrm{A}_{1}\right),\left(\mathrm{A}_{2}\right)$ and $\left(\mathrm{A}_{3}\right)$ hold. Let $\left\{u_{j}(x, t)\right\}(j=1, \ldots, N)$ be a solution of $(1 \mathrm{~h})$ in $R_{n} \times(0, T]$ such that

$$
\left\|u_{j}(\cdot, t) \exp \left(-\alpha|\cdot|^{2 m /(2 m-1)}\right)\right\|_{L\left(R_{n}\right)}<M \quad(j=1, \ldots, N)
$$

for all $0<t \leqslant T$, where $M$ and $\alpha$ are positive constants, $1<p<\infty$. Then there exist unique functions $f_{j}(j=1, \ldots, N)$ such that $f_{j} \exp \left(-\alpha|x|^{2 m /(2 m-1)}\right) \in$ $L^{p}\left(R_{n}\right)(j=1, \ldots, N)$ and

$$
u_{i}(x, t)=\int_{R_{n} j=1} \sum_{i j}^{N} \Gamma_{i j}(x, t ; y, 0) f_{j}(y) d y \quad(i=1, \ldots, N)
$$

for $(x, t) \in(0, T] \times R_{n}$.

The case $p=1$ is not contained in Theorem 2.2, but using a similar argument one can prove

THEOREM 2.3. Suppose that the hypotheses $\left(\mathrm{A}_{1}\right),\left(\mathrm{A}_{2}\right)$ and $\left(\mathrm{A}_{3}\right)$ hold. Let $\left\{u_{j}(x, t)\right\}(i=1, \ldots, N)$ be a solution of the system $(1 \mathrm{~h})$ in $R_{n} \times(0, T]$ such that

$$
\left\|u_{i}(\cdot, t) \exp \left(-\alpha|\cdot|^{2 m /(2 m-1)}\right)\right\|_{L^{1}\left(R_{n}\right)}<M \quad(i=1, \ldots, N)
$$

for all $0<t \leqslant M$, where $\alpha$ and $M$ are positive constants. Then there exist unique Borel measures $\left\{\mu_{j}\right\}(j=1, \ldots, N)$ such that

$$
\int_{R_{n}} \exp \left(-\alpha|x|^{2 m /(2 m-1)}\right)\left|\mu_{j}\right|(d x)<\infty \quad(i=1, \ldots, N)
$$

and

$$
u_{i}(x, t)=\int_{R_{n}} \sum_{j=1}^{N} \Gamma_{i j}(x, t ; y, 0) \mu_{j}(d y) \quad(i=1, \ldots, N)
$$

for all $(x, t) \in R_{n} \times(0, T]$.

\section{Global Fatou property}

We shall derive an analogue of the well known Fatou property for bounded harmonic functions in an open disc. Roughly speaking, this property asserts that a bounded harmonic function in an open disc admits a non-tangential limit 
almost everywhere on the boundary. In this section we establish the existence of a parabolic limit for solutions of the system (1h).

Given $\gamma>0, h>0$ and a point $x_{0} \in R_{n}$, let

$$
\Gamma_{\gamma}^{h}\left(x_{0}\right)=\left\{(x, t) \in R_{n+1} ; 0<\left|x-x_{0}\right|<\gamma t^{1 /(2 m)}, 0<t<h\right\}
$$

denote a truncated parabolic region in $R_{n+1}$.

We shall say that a function $u$ defined on $R_{n} \times(0, h]$ has a parabolic limit at $x_{0}$ if there exists a number 1 such that for all $\gamma>0$, we have

$$
\lim _{\substack{(x, t) \rightarrow\left(x_{0}, 0\right) \\(x, t) \in \Gamma_{\gamma}^{\mathrm{h}}\left(x_{0}\right)}} u(x, t)=1,
$$

We shall briefly express this by writing $\mathrm{p}-\lim _{(x, t) \rightarrow\left(x_{0}, 0\right)} u(x, t)=1$.

Further, given a Borel measure $\mu$ on $R_{n}$ and a point $x \in R_{n}$, we define the symmetric derivative $D \mu$ of $\mu$ at $x$, by

$$
D \mu(x)=\lim _{\delta \rightarrow 0} \frac{\mu(B(x, \delta))}{m(B(x, \delta))},
$$

where $m$ denotes the Lebesgue measure on $R_{n}$, and $B(x, \delta)=\{y ;|y-x|<\delta\}$. It is known that $D \mu$ exist a.e. $[m]$.

THEOREM 3.1. Suppose that $\left(\mathrm{A}_{1}\right)$ and $\left(\mathrm{A}_{2}\right)$ hold. Let $f_{j} \in \tilde{L}_{\Phi}^{\alpha}\left(R_{n}\right) \quad(j=$ $1, \ldots, N)$ and let $\left\{u_{i}(x, t)\right\}(i=1, \ldots, N)$ be a solution of $(\mathrm{lh})$ in $R_{n} \times(0, T]$ given by

$$
u_{i}(x, t)=\int_{R_{n} j} \sum_{j=1}^{N} \Gamma_{i j}(x, t ; y, 0) f_{j}(y) d y \quad(i=1, \ldots, N),
$$

then

$$
\operatorname{pim}_{(x, t) \rightarrow\left(x_{0}, 0\right)} u_{i}(x, t)=f_{i}\left(x_{0}\right) \quad(i=1, \ldots, N)
$$

exists a.e. $[m]$ in $R_{n}$. Moreover, if $\left\{\mu_{j}\right\}(j=1, \ldots, N)$ are Borel measures satisfying condition $(20)$ and

$$
u_{i}(x, t)=\int_{R_{n}} \sum_{j=1}^{N} \Gamma_{i j}(x, t ; y, 0) \mu_{j}(d y) \quad(i=1, \ldots, N),
$$

then

$$
\operatorname{p}_{(x, t) \rightarrow\left(x_{0}, 0\right)} u_{i}(x, t)=D \mu_{i}\left(x_{0}\right) \quad(i=1, \ldots, N)
$$

exists a.e. $[m]$ in $R_{n}$.

Proof. It suffices to prove the second part of the theorem. Write the Lebesgue decomposition of the measures

$$
\mu_{j}(d y)=g_{j}(y) d y+v_{j}(d y) \quad(j=1, \ldots, N),
$$


where the functions $g_{j}$ belong (locally) to $L^{1}\left(R_{n}\right)$ and $\nu_{j}$ are singular with respect to the Lebesgue measure. We then have that

$$
\lim _{a \rightarrow 0} \frac{1}{a^{n}} \int_{|y-x|<a}\left[\left|g_{j}(y)-g_{j}(x)\right| d y+\left|\nu_{j}\right|(d y)\right]=0 \quad(j=1, \ldots, N)
$$

a.e. [ $m$ ] in $R_{n}$. Hence for fixed $x$ and (for any) $\varepsilon>0$ there exists a number $\delta>0$ such that

$$
a^{-n} \int_{|y-x|<a}\left[\left|g_{j}(y)-g_{j}(x)\right| d y+\left|y_{j}\right|(d y)\right]<\varepsilon \quad(j=1, \ldots, N)
$$

for all $0<a \leqslant 2 \delta$. For each $0<t \leqslant \min (2 \delta, T)$ choose a non-negative integer $P(t)$ such that

$$
2^{P-1} \gamma t^{1 /(2 m)}<\delta<2^{P} \gamma t^{1 /(2 m)}
$$

It is clear that

$$
\begin{aligned}
& \left|u_{i}(z, t)-\int_{R_{n} j=1} \sum_{i j}^{N} \Gamma_{i j}(z, t ; y, 0) g_{i}(x) d y\right| \\
& \leqslant \sum_{j=1}^{N} \int_{|y-x|<t^{1 / 2 m}}\left|\Gamma_{i j}(z, t ; y, 0)\right|\left[\left|g_{j}(y)-g_{j}(x)\right| d y+\left|v_{j}\right|(d y)\right] \\
& +\sum_{l=2}^{P} \int_{2^{1-1} y t^{1 / 2 m)}<|y-x|<Z\left\langle t^{1 /(2 m)}\right)} \sum_{j=1}^{N}\left|\Gamma_{i j}(z, t ; y, 0)\right|\left[\left|g_{j}(y)-g_{j}(x)\right| d y+\left|\nu_{j}\right|(d y)\right] \\
& +\sum_{j=1}^{N} \int_{|y-x|>\delta}\left|\Gamma_{i j}(z, t ; y, 0)\right|\left|g_{j}(x)\right| d y+\sum_{j=1}^{N} \int_{|y-x|>\delta}\left|\Gamma_{i j}(z, t ; y, 0)\right|\left|\mu_{j}\right|(d y) \\
& =J_{1}+J_{2}+J_{3}+J_{4} \text {. }
\end{aligned}
$$

In view of (22) we have

$$
\begin{aligned}
J_{1} \leqslant C \int_{|y-x|<2 \gamma t^{1 /(2 m)}} t^{-n /(2 m)} \exp \left[-x\left(\frac{|y-x|^{2 m}}{t}\right)^{1 /(2 m-1)}\right] \\
\times\left[\sum_{j=1}^{N}\left|g_{j}(y)-g_{j}(x)\right| d y+\left|\nu_{j}\right|(d y)\right]<N C E(2 \gamma)^{n} .
\end{aligned}
$$

Note that the inequalities $|z-x|<\gamma t^{1 /(2 m)}$ and $|x-y|>2^{l-1} \gamma t^{1 /(2 m)}$ imply $|z-y| \geqslant 2^{(l-2)} \gamma t^{1 /(2 m)}$ for $l=2, \ldots, P$ hence

$$
\begin{aligned}
J_{2} & \leqslant \sum_{l=2}^{P} N C \varepsilon 2^{n l} \gamma^{n} \exp \left[-\chi\left(2^{l-2} \gamma\right)^{2 m /(2 m-1)}\right] \\
& \leqslant C N \varepsilon \sum_{l=2}^{\infty} 2^{l n} \gamma^{n} \exp \left[-\chi\left(2^{l-2} \gamma\right)^{2 m /(2 m-1)}\right] .
\end{aligned}
$$

Now using the estimate (5) and the condition (20) it is easy to show that $\mathrm{p}-\lim _{(2, t) \rightarrow(x, 0)} J_{i}=0, i=3,4$. 
Finally, noting that

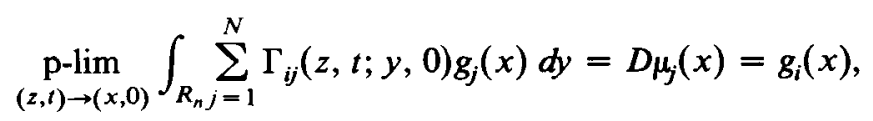

the result follows.

\section{Parabolic systems of second order, non-negativity of the fundamental matrix}

The purpose of this section is to formulate a necessary and sufficient condition for the fundamental matrix to have non-negative elements. We obtain the result that a parabolic system of the second order having non-negative elements in its matrix must be weakly coupled.

We shall first establish theorems dealing with changes of sign of the elements of the fundamental matrix.

Let us consider the second order system of partial differential equations of the form

$$
\frac{\partial u_{i}}{\partial t}=\sum_{j=1}^{N} \sum_{|k|<2} A_{k}^{i j}(x, t) D_{x}^{k} u_{j} \quad(i=1, \ldots, N)
$$

on the strip $R_{n} \times(0, T]$.

Introduce the following notation. By $k^{m l}$ we denote the sequence $\left(k_{1}, \ldots, k_{n}\right)$ of non-negative integers such that

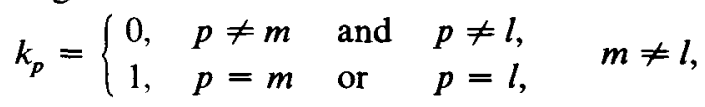

$k^{m m}$ denotes the sequence $\left(2 \delta_{j m}\right)(j=1, \ldots, n)$ and finally $k^{m}$ denotes the sequence $\left(\delta_{j m}\right)(j=1, \ldots, n)$.

Set $A_{0}^{i j}(x, t)=A_{k}^{i j}(x, t)$, whenever $|k|=0, i, j=1, \ldots, N$.

We shall need the following lemma proved by Milicer-Grużewska (1960), (1963).

LEMMA 4.1. Suppose that $\left(\mathrm{A}_{1}\right),\left(\mathrm{A}_{2}\right)$ and $\left(\mathrm{A}_{3}\right)$ hold. Then for every $(x, t) \in R_{n} \times$ $[0, T]$ we have

$$
\begin{gathered}
\lim _{\tau \rightarrow t} \frac{1}{2(t-\tau)} \int_{R_{n}} \Gamma_{i j}(x, t ; y, \tau)\left(y_{m}-x_{m}\right)\left(y_{l}-x_{l}\right) d y=A_{k^{m}}^{i j}(x, t), \\
\lim _{\tau \rightarrow t} \frac{1}{2(t-\tau)} \int_{R_{n}} \Gamma_{i j}(x, t ; y, \tau)\left(y_{m}-x_{m}\right)^{2} d y=A_{k^{m}}^{i j}(x, t), \\
\lim _{\tau \rightarrow t} \frac{1}{t-\tau} \int_{R_{n}} \Gamma_{i j}(x, t ; y, \tau)\left(y_{m}-x_{m}\right) d y=A_{k^{m}}^{i j}(x, t), \\
\lim _{\tau \rightarrow t} \frac{1}{t-\tau} \int_{R_{n}} \Gamma_{i j}(x, t ; y, \tau) d y=A_{0}^{i j}(x, t), \quad m, l=1, \ldots, n, i \neq j .
\end{gathered}
$$


LEMMA 4.2. Let $\left(\mathrm{A}_{1}\right),\left(\mathrm{A}_{2}\right)$ and $\left(\mathrm{A}_{3}\right)$ hold. Then for every function $\phi \in C^{3}\left(R_{n}\right)$ (with bounded derivatives of the third order) we have

$$
\lim _{\tau \rightarrow t} \frac{1}{t-\tau} \int_{R_{n}} \Gamma_{i j}(x, t ; y, \tau) \phi(y) d y=\sum_{|k|<2} A_{k}^{i j}(x, t) D_{x}^{k} \phi(x)
$$

for $i \neq j, x \in R_{n}$.

The result follows easily from Lemma 4.1 and Taylor's formula.

On the basis of the above lemma, we can now establish the following result.

THEOREM 4.1. Suppose the hypotheses $\left(\mathrm{A}_{1}\right),\left(\mathrm{A}_{2}\right)$ and $\left(\mathrm{A}_{3}\right)$ hold. If for fixed $i \neq j$ and multi-index $(k),|k|=2, A_{k}^{i j}(x, t) \neq 0$, then the element $\Gamma_{i j}(x, t ; y, \tau)$ of the fundamental matrix changes sign.

Proof. Let us consider the case $(k)=\left(k^{m m}\right)$. According to the assumption there exists a point $\left(x^{\prime}, t^{\prime}\right)$ such that $A_{k=m}^{i j}\left(x^{\prime}, t^{\prime}\right) \neq 0$. Let $A_{k=m}^{i j m}\left(x^{\prime}, t^{\prime}\right)>0$. If we assume, contrary to the theorem, that $\Gamma_{i j} \leqslant 0$, then by Lemma 4.1

$$
\lim _{\tau \rightarrow t^{\prime}} \frac{1}{2\left(t^{\prime}-\tau\right)} \int_{R_{n}} \Gamma_{i j}\left(x^{\prime}, t^{\prime} ; y, \tau\right)\left(y_{m}-x_{m}^{\prime}\right)^{2} d y=A_{k-m}^{i j}\left(x^{\prime}, t^{\prime}\right)<0,
$$

which is impossible. Similarly, if $\Gamma_{i j}$ is non-negative, then by Lemma 4.2 we have in the case $A_{0}^{i j}\left(x^{\prime}, t^{\prime}\right) \leqslant 0$

$$
\begin{gathered}
\lim _{\tau \rightarrow t} \frac{1}{t^{\prime}-\tau} \int_{R_{n}} \Gamma_{i j}\left(x^{\prime}, t^{\prime} ; y, \tau\right)\left[1+\cos ^{2}\left(y_{m}-x_{m}^{\prime}\right)\right] d y \\
=2 A_{0}^{i j}\left(x^{\prime}, t^{\prime}\right)-2 A_{k}^{i j}\left(x^{\prime}, t^{\prime}\right)<0
\end{gathered}
$$

and in the case $A_{0}^{i j}\left(x^{\prime}, t^{\prime}\right)>0$

$$
\lim _{\tau \rightarrow t^{\prime}} \frac{1}{t^{\prime}-\tau} \int_{R_{j}} \Gamma_{i j}\left(x^{\prime}, t^{\prime} ; y, \tau\right) f\left(y_{m}\right) d y=-A_{k^{m m}}^{i j}\left(x^{\prime}, t^{\prime}\right)>0,
$$

where

$$
f\left(y_{m}\right)=\left[\frac{A_{k^{m m}}^{i j}\left(x^{\prime}, t^{\prime}\right)}{A_{0}^{i j}\left(x^{\prime}, t^{\prime}\right)}-\left(y_{m}-x_{m}^{\prime}\right)^{2}\right] \phi\left(y_{m}\right) .
$$

Here $\phi\left(y_{m}\right)$ is a cut-off function such that $\phi \in C^{3}(R), 0<\phi<1, \phi\left(y_{m}\right)=1$ for $\left|x_{m}^{\prime}-y_{m}\right|<\delta / 2, \phi\left(y_{m}\right)=0$ for $\left|x_{m}^{\prime}-y_{m}\right| \geqslant \delta$ and a positive $\delta$ is chosen such that

$$
\frac{A_{k^{m m}}^{i j}\left(x^{\prime}, t^{\prime}\right)}{A_{0}^{i j}\left(x^{\prime}, t^{\prime}\right)}-\left(y_{n}-x_{m}^{\prime}\right)^{2}>0
$$


for $\left|y_{m}-x_{m}^{\prime}\right|<\delta$. It is clear that in both cases we have arrived at the contradiction. Clearly, the same method can be applied in the case $A_{k^{m m}}^{i j}\left(x^{\prime}, t^{\prime}\right)$ $<0$ and subsequently when $(k)=\left(k^{m l}\right)$.

Using the above technique we may prove

THEOREM 4.2. Suppose the hypotheses $\left(\mathrm{A}_{1}\right),\left(\mathrm{A}_{2}\right)$ and $\left(\mathrm{A}_{3}\right)$ hold. If for fixed $i \neq j$ and multi-index $(k),|k|=1, A_{k}^{i j}(x, t) \not \neq 0$, then the element $\Gamma_{i j}$ changes sign.

As an immediate consequence of Theorems 4.1 and 4.2 we obtain

COROllary 4.1. Let $\left(\mathrm{A}_{1}\right),\left(\mathrm{A}_{2}\right)$ and $\left(\mathrm{A}_{3}\right)$ hold. If for fixed $i \neq j$ and multi-index (k) with $|k|=2$ or $|k|=1, A_{k}^{i j}(x, t) \neq 0$, then the element $\Gamma_{i j}$ changes sign.

To prove the main result of this section we shall need the following maximum principle, due to Besala (1972), for weakly coupled parabolic systems:

$$
\sum_{|k|=1,2} A_{k}^{i i}(x, t) D_{x}^{k} u_{i}+\sum_{j=1}^{N} A_{0}^{i j}(x, t) u_{j}-\frac{\partial u_{i}}{\partial t}=0 \quad(i=1, \ldots, N) .
$$

By $L_{i}$ we denote the operator which is defined by the left-hand side of (24).

It is easily seen that parabolicity in the sense of Petrowskii means that

(P) there is a positive number $\delta$ such that

$$
\sum_{i, j=1}^{n} A_{k_{j}}^{r r}(x, t) \xi_{i} \xi_{j} \geqslant \delta|\xi|^{2} \quad(r=1, \ldots, N)
$$

for all $\xi \in R_{n},(x, t) \in R_{n} \times[0, T]$.

THEOREM 4.3. Let the coefficients of the system (24) satisfy the assumptions $\left(\mathrm{A}_{1}\right)$, $\left(\mathrm{A}_{2}\right),\left(\mathrm{A}_{3}\right)$ and $(\mathrm{P})$ and moreover let

$$
A_{0}^{i j}(x, t) \geqslant 0 \text { for all }(x, t) \in R_{n} \times[0, T], i \neq j .
$$

Assume that the functions $\left\{u_{i}(x, t)\right\}(i=1, \ldots, N)$ satisfy the differential inequalities

$$
L_{i}\left(u_{1}, \ldots, u_{n}\right) \leqslant 0 \quad(i=1, \ldots, N)
$$

in $R_{n} \times(0, T]$, are continuous on $R_{n} \times[0, T]$, and

$$
\int_{0}^{T} \int_{R_{n}} u_{i}^{-}(x, t) \exp \left(-b|x|^{2}\right) d x d t<\infty \quad(i=1, \ldots, N),
$$

where $b>0, u_{i}^{-}(x, t)=\max \left(0,-u_{i}(x, t)\right)$ and

$$
u_{i}(x, 0) \geqslant 0 \quad(i=1, \ldots, N)
$$


for all $x \in R_{n}$, then

$$
u_{i}(x, t) \geqslant 0 \quad(i=1, \ldots, N)
$$

for all $(x, t) \in R_{n} \times[0, T]$,

THEOREM 4.4. Assume that the system (23) is uniformly parabolic in $R_{n} \times[0, T]$ and that $\left(\mathrm{A}_{1}\right),\left(\mathrm{A}_{2}\right)$ and $\left(\mathrm{A}_{3}\right)$ hold in $R_{n} \times[0, T]$. All elements $\Gamma_{i j}(x, t ; y, \tau)$ of the fundamental matrix of the system (23) are non-negative if and only if

$$
A_{k}^{i j}(x, t) \equiv 0, \quad|k|=1,2, i \neq j,
$$

and

$$
A_{0}^{i j}(x, t) \geqslant 0, \quad i \neq j,
$$

for all $(x, t) \in R_{n} \times[0, T]$.

Proof. First, let us suppose that (26) and (27) hold. Then the system (23) takes the form (24). Fix an index $i(i=1, \ldots, N)$ and consider the Cauchy problem for the system (24) with the initial data

$$
u_{j}(x, \tau)=\delta_{i j} \phi(x) \quad(j=1, \ldots, N),
$$

where $\phi$ is a continuous, non-negative bounded function in $R_{n}$. Theorem 1.2 (formula (8)) implies that the solution of the Cauchy problem (24), (28) is given by the formula

$$
u_{j}(x, t)=\int_{R_{n}} \Gamma_{j i}(x, t ; y, \tau) \phi(y) d y \quad(j=1, \ldots, N) .
$$

By Theorem 4.3

$$
\int_{R_{n}} \Gamma_{j i}(x, t ; y, \tau) \phi(y) d y>0 \quad(j=1, \ldots, N)
$$

for any non-negative continuous bounded function $\phi$, hence $\Gamma_{j i}(x, t ; y, \tau)>0$ for $(x, t),(y, \tau) \in R_{n} \times[0, T], \tau<t$.

To prove the converse assume that $\Gamma_{i j}(x, t ; y, \tau)>0$ for $(x, t),(y, \tau) \in R_{n} \times$ $[0, T], t>\tau$. Then Corollary 4.1 implies (26). The non-negativity of $A_{0}^{i j}(x, t)$ is a consequence of Lemma 4.1.

Remark 4.1. It follows from Theorem 4.4 that the fundamental solution of a single parabolic equation of the second order is nonnegative. However the non-negativity of the fundamental solution will break down for a parabolic equation of higher order. As an example, due to Kondrat'ev and Eidel'man (1974), consider the parabolic equation with constant coefficients

$$
\frac{\partial u}{\partial t}=\sum_{|k|=2 m} A_{k} D_{x}^{k} u, \quad m>1 .
$$


Using the Fourier transform we easily find that the fundamental solution is given by

$$
\Gamma(x, t)=(2 \pi)^{-n} \int_{R_{n}} \exp \left[i \sum_{j=1}^{N} x_{j} y_{j}+(-1)^{m} \sum_{|k|=2 m} A_{k} y^{k} t\right] d y .
$$

It follows directly from the above formula that $\Gamma(0, t)>0$. Let

$$
u(x, t)=\int_{R_{n}} \Gamma(x-y, t) \phi(y) d y,
$$

where $\phi$ is a non-negative infinitely differentiable function equal to $x_{1}^{2}+$ $(-1)^{m} x_{1}^{2 m}$ in a neighborhood of the origin. Then $u(x, t)$ is also infinitely differentiable right up to the hyperplane $t=0$. Calculating the derivative in $t$ and using the parabolicity condition we obtain

$$
\begin{aligned}
\left.\frac{\partial u}{\partial t}\right|_{x=0, t=0} & =\left.\sum_{|k|=2 m} A_{k} D_{x}^{k} u\right|_{x=0, t=0}=\left.\sum_{|k|=2 m} A_{k} D_{x}^{k} \phi(x)\right|_{x=0} \\
& =(2 m) !(-1)^{m} A_{2 m, 0, \ldots, 0}<0 .
\end{aligned}
$$

But $u(0,0)=0$ and hence $u(0, t)>0$ for sufficiently small $t$, which is impossible if $\Gamma(x, t) \geqslant 0$.

We conclude this section by finding a lower bound of the fundamental solution of the weakly coupled parabolic system (24). Fix an index $i$ and let $\Gamma_{i}(x, t ; y, \tau)$ be the fundamental solution the parabolic equations

$$
\frac{\partial v}{\partial t}=\sum_{0<|k|<2} A_{k}^{i i}(x, t) D_{x}^{k} v \text {. }
$$

THEOREM 4.5. Suppose that the coefficients of the system (24) satisfy the assumptions $\left(\mathrm{A}_{1}\right),\left(\mathrm{A}_{2}\right),\left(\mathrm{A}_{3}\right),(\mathrm{P})$ and $(27)$. Let $\Gamma_{i}(x, t ; y, \tau)(i=1, \ldots, N)$ be the fundamental solution of (29). Then

$$
\Gamma_{i}(x, t ; y, \tau) \leqslant \Gamma_{i i}(x, t ; y, \tau) \quad(i=1, \ldots, N)
$$

for all $(x, t),(y, \tau) \in R_{n} \times[0, T], \tau<t$.

Proof. Let $\phi$ be a bounded, non-negative, continuous function on $R_{n}$ and set

$$
r_{i}(x, t)=\int_{R_{n}}\left[\Gamma_{i i}(x, t ; y, \tau)-\Gamma_{i}(x, t ; y, \tau)\right] \phi(y) d y .
$$

By (4) we have $\lim _{t \rightarrow \tau} r_{i}(x, t)=0(i=1, \ldots, N)$. Note that $r_{i}$ satisfies the equation

$$
\sum_{0<|k|<2} A_{k}^{i i}(x, t) D_{x}^{k} r_{i}-\frac{\partial r_{i}}{\partial t}=-\sum_{j \neq i} A_{0}^{i j}(x, t) \int_{R_{n}} \Gamma_{i j}(x, t ; y, \tau) \phi(y) d y .
$$


Since the right hand side of the last equation is non-positive it follows from Theorem 4.3 that $r_{i}(x, t) \geqslant 0$ for $(x, t) \in R_{n} \times(\tau, T](i=1, \ldots, N)$ and consequently

$$
\int_{R_{n}} \Gamma_{i}(x, t ; y, \tau) \phi(y) d y \leqslant \int_{R_{n}} \Gamma_{i i}(x, t ; y, \tau) \phi(y) d y
$$

for any non-negative, continuous, bounded function on $R_{n}$, and the proof is complete.

COROLLARY 4.2. Under the assumptions of Theorem 4.5 we have

$$
C_{1}(t-\tau)^{-n / 2} \exp \left(-\chi_{1} \frac{|x-y|^{2}}{t-\tau}\right)<\Gamma_{i i}(x, t ; y, \tau) \quad(i=1, \ldots, N)
$$

for all $(x, t),(y, \tau) \in R_{n} \times[0, T], \tau<t$, where $C_{1}$ and $\chi_{1}$ are positive constants.

The result follows from (30) and the lower estimate for $\Gamma_{i}$ established by Aronson (1968)

$$
C_{1}(t-\tau)^{-n / 2} \exp \left(-\chi_{1} \frac{|x-y|^{2}}{t-\tau}\right) \leqslant \Gamma_{i}(x, t ; y, \tau)
$$

for $(x, t),(y, \tau) \in R_{n} \times[0, T], \tau<t$.

\section{Representation of non-negative solutions of weakly coupled parabolic systems}

Theorem 4.4 shows that the investigation of non-negative solutions makes sense only for weakly coupled parabolic system (24), whose coefficients $A_{0}^{i j}$ are non-negative for $i \neq j$.

Throughout this section it will be assumed that the system (24) is uniformly parabolic and satisfies $\left(A_{1}\right),\left(A_{2}\right),\left(A_{3}\right)$ and $(27)$.

THEOREM 5.1. Let $\left\{u_{i}(x, t)\right\}(i=1, \ldots, N)$ be a non-negative solution of (24) in $R_{n} \times(0, T]$. Then there are unique non-negative Borel measures $\left\{\mu_{j}\right\}(j=$ $1, \ldots, N)$ such that

$$
\begin{gathered}
u_{i}(x, t)=\int_{R_{n} j=1} \sum_{i j}^{N} \Gamma_{i j}(x, t ; y, 0) \mu_{j}(d y), \\
\int_{R_{n}} \exp \left(-\beta|x|^{2}\right) \mu_{i}(d x)<\infty \quad(i=1, \ldots, N)
\end{gathered}
$$

for $(x, t) \in R_{n} \times(0, T]$, where $\beta$ is a positive constant. 
PROOF. Let $h_{R}(x)$ be a non-negative cut-off function such that $0<h_{R}(x)<1$ in $R_{n}, h_{R}(x)=1$ for $|x| \leqslant R$, and $h_{R}(x)=0$ for $|x|>R+1$. For fixed $\tau \in$ $[0, T)$ consider the Cauchy problem for the system (24) in $R_{n} \times(\tau, T]$ with the initial data

$$
v_{i}(x, \tau)=h_{R}(x) u_{i}(x, \tau) \quad(i=1, \ldots, N)
$$

for $x \in R_{n}$. It follows from Theorem 1.2 that

$$
v_{i}(x, t)=\int_{R_{n} j=1} \sum_{i j}^{N} \Gamma_{i j}(x, t ; y, \tau) h_{R}(y) u_{j}(y, \tau) d y \quad(i=1, \ldots, N)
$$

for $(x, t) \in R_{n} \times(\tau, T]$. Note that functions $\left\{u_{i}(x, t)-v_{i}(x, t)\right\} \quad(i=$ $1, \ldots, N)$ are bounded below and

$$
\lim _{t \rightarrow \tau}\left[u_{i}(x, t)-v_{i}(x, t)\right]=u_{i}(x, \tau)\left(1-h_{R}(x)\right)>0 \quad(i=1, \ldots, N)
$$

for $x \in R_{n}$. By Theorem 4.3

$$
u_{i}(x, t) \geqslant v_{i}(x, t) \quad(i=1, \ldots, N),
$$

for $(x, t) \in R_{n} \times(\tau, T]$. The last inequality can be written in the form

$$
\int_{R_{n} j=1} \sum_{i j}^{N} \Gamma_{i j}(x, t ; y, \tau) u_{j}(y, \tau) h_{R}(y) d y<u_{i}(x, t) \quad(i=1, \ldots, N)
$$

Letting $R \rightarrow \infty$ and using (30) we obtain

$$
\int_{R_{n}} \Gamma_{i}(x, t ; y, \tau) u_{i}(y, \tau) d y \leqslant \int_{R_{n}} \Gamma_{i j}(x, t ; y, \tau) u_{i}(y, \tau) d y<u_{i}(x, t)
$$

$(i=1, \ldots, N)$, for $(x, t) \in R_{n} \times(\tau, T]$. Now set $(x, t)=(0, T)$ and let $0<\tau$ $<T_{1}$, where $T_{1}<T$. It follows from Corollary 4.2 that

$$
\int_{R_{n}} \exp \left(-\frac{x_{1}}{T-T_{1}}|y|^{2}\right) u_{i}(y, \tau) d y \leqslant T^{n / 2} C_{1}^{-1} u_{i}(0, T) \quad(i=1, \ldots, N)
$$

for $0<\tau<T_{1}$. The representation formulae (31) and (32) in $R_{n} \times\left(0, T_{1}\right]$ are the immediate consequence of Theorem 2.3. The Kolmogorov identity allows the extension of the validity of his representation formula to $R_{n} \times(0, T]$.

Corollary 5.1. Under the assumption of Theorem $5.1 \mu_{i}(d x)=u_{i}(x, 0) d x$ $(i=1, \ldots, N)$ provided the solution $\left\{u_{i}(x, t)\right\}(i=1, \ldots, N)$ is continuous on $\boldsymbol{R}_{n} \times[0, T]$.

We shall now extend Theorems 2.2 and 2.3 to the solutions of (24) satisfying the growth condition

$$
\int_{R_{n}} \Phi\left(u_{j}^{-}(x, t)\right) \exp \left(-\alpha|x|^{2}\right) d x<M \quad(i=1, \ldots, N)
$$


for all $0<t \leqslant T$, where $\Phi$ denotes a Young function, $\alpha$ and $M$ are positive constants.

THEOREM 5.2. Let $\left\{u_{i}(x, t)\right\}(i=1, \ldots, N)$ be a solution of $(24)$ in $R_{n} \times(0, T]$ such that (33) holds.

Then there exist functions $f_{j} \in \tilde{L}_{\Phi}^{\alpha}\left(R_{n}\right)(j=1, \ldots, N)$ and non-negative Borel measures $\mu_{j}(j=1, \ldots, N)$ such that

$$
\int_{R_{n}} \exp \left(-\beta|x|^{2}\right)(d x)<\infty \quad(j=1, \ldots, N),
$$

where $\beta$ is a positive constant, and

$$
\begin{aligned}
u_{i}(x, t)=\int_{R_{n}} \sum_{j=1}^{N} \Gamma_{i j}(x, t ; y, 0) \mu_{j}(d y)-\int_{R_{n}} \sum_{j=1}^{N} \Gamma_{i j}(x, t ; y, 0) f_{j}(y) d y & \\
& (i=1, \ldots, N)
\end{aligned}
$$

for all $(x, t) \in R_{n} \times(0, T]$.

Proof. The proof is similar to that of Theorem 2.2, so we shall merely sketch the details. By the estimates (5) and Theorem 4.3 there is a constant $\delta>0$ independent of $\tau$ such that

$$
u_{i}(x, t)+\int_{R_{n}} \sum_{j=1}^{N} \Gamma_{i j}(x, t ; y, \tau) u_{j}^{-}(y, \tau) d y>0 \quad(i=1, \ldots, N)
$$

for $(x, t) \in R_{n} \times(\tau, \min (T, \tau+\delta)]$. On the other hand repeating the argument of Theorem 2.2 there exist a sequence $t_{v}$ converging to 0 and functions $f_{j} \in$ $\tilde{L}_{\Phi}^{\alpha}\left(R_{n}\right)$ such that

$$
\begin{aligned}
\lim _{\nu \rightarrow \infty} \int_{R_{n}} \sum_{j=1}^{N} \Gamma_{i j}\left(x, t ; y, t_{\nu}\right) u_{j}^{-}\left(y, t_{\nu}\right) d y=\int_{R_{n}} \sum_{j=1}^{N} \Gamma_{i j}(x, t ; y, 0) f_{j}(y) d y & \\
& (i=1, \ldots, N) .
\end{aligned}
$$

Now the result follows from Theorem 5.1.

Similarly one can prove

THEOREM 5.3. Let $\left\{u_{i}(x, t)\right\}(i=1, \ldots, N)$ be a solution of the system $(24)$ in $R_{n} \times(0, T]$ such that

$$
\int_{R_{n}} u_{j}^{-}(x, t) \exp \left(-\alpha|x|^{2}\right) d x<M \quad(i=1, \ldots, N)
$$

for all $t \in(0, T]$, where $M$ and $\alpha$ are positive constants. 
Then there exist signed measures $\left\{\mu_{j}\right\}(j=1, \ldots, N)$ and nonnegative Borel measures $\left\{\nu_{j}\right\}(j=1, \ldots, N)$ satisfying $(20)($ with $m=1)$ such that

$$
u_{i}(x, t)=\int_{R_{n}} \sum_{j=1}^{N} \Gamma_{i j}(x, t ; y, 0) \mu_{j}(d y)-\int_{R_{n} j=1}^{N} \Gamma_{i j}(x, t ; y, 0) \nu_{j}(d y)
$$

$$
(i=1, \ldots, N)
$$

for all $(x, t) \in R_{n} \times(0, T]$

\section{Differentiation of measures and initial values of non-negative solutions}

First we state some facts on differentiation of Borel measures, due to Watson (1977), which play an essential part in the treatment of the pointwise estimation of non-negative solutions.

For each Borel measure $\mu$ on $R_{n}$ and each point $x \in R_{n}$, we define the upper symmetric derivative $(\bar{D} \mu)(x)$ of $\mu$ at $x$ by

$$
(\bar{D} \mu)(x)=\limsup _{\delta \rightarrow 0} \frac{\mu(B(x, \delta))}{m(B(x, \delta))},
$$

and the lower symmetric derivative $(\underline{D} \mu)(x)$ of $\mu$ at $x$ by

$$
(\underline{D} \mu)(x)=\liminf _{\delta \rightarrow 0} \frac{\mu(B(x, \delta))}{m(B(x, \delta))},
$$

where $m$ denotes the Lebesgue measure on $R_{n}$. When $(\underline{D} \mu)(x)=(\bar{D} \mu)(x)$ we say that the symmetric derivative of $\mu$ at $x$ exists and denote it by $(D \mu)(x)$ (see also Section 3).

Lemma 6.1. Suppose that $\mu$ is a positive measure on $R_{n}$, and that $\mu$ is singular with respect to the Lebesgue measure. Then $(D \mu)(x)=\infty$ a.e. $[\mu]$.

LEMMA 6.2. Let $\mu$ be a positive measure on $R_{n}$. If $(D \mu)(x)<\infty$ for all $x$ at which it exists, then $\mu$ is absolutely continuous with respect to the Lebesgue measure.

LEMMA 6.3. If $\mu$ is a positive measure on $R_{n}$ and $\left(\bar{D}_{\mu}\right)(x)<\infty$ for all $x \in R_{n}$, then $\mu$ is absolutely continuous with respect to the Lebesgue measure.

Suppose that $\nu$ is a positive Borel measure such that

$$
\int_{R_{n}} \exp \left(-a|x|^{2}\right) \nu(d x)<\infty
$$


for some positive constant $a$. Let $\rho$ be a positive constant and define

$$
v_{\rho, \nu}(x, t)=t^{-n / 2} \int_{R_{n}} \exp \left(-\rho \frac{|x-y|^{2}}{t}\right) \nu(d y) .
$$

It follows from (34) that $v_{\rho, v}$ is finite for $t$ sufficiently small.

LEMMA 6.4. Let $\mu$ and $\nu$ be a positive Borel measure satisfying (34) and let $x \in R_{n}$. If there exists $\delta>0$ such that $\nu(B(x, r))>0$ for $r \in(0, \delta]$, then

$$
\begin{aligned}
\liminf _{r \rightarrow 0} \frac{\mu(B(x, r))}{\nu(B(x, r))} & <\liminf _{t \rightarrow 0} \frac{v_{\rho, \mu}(x, t)}{v_{\delta, \nu}(x, t)} \leqslant \lim \sup _{t \rightarrow 0} \frac{v_{\rho, \mu}(x, t)}{v_{\delta, \nu}(x, t)} \\
& <\limsup _{r \rightarrow 0} \frac{\mu(B(x, r))}{\nu(B(x, r))} .
\end{aligned}
$$

Proof. It is easy to show that for every $\varepsilon>0$

$$
\int_{|x-y|>\varepsilon} t^{-n / 2} \exp \left(-\rho \frac{|x-y|^{2}}{t}\right) \mu(d y)=o\left(v_{\rho, \nu}(x, t)\right)
$$

as $t \rightarrow 0$. We shall prove only the first inequality. Let $\lambda$ be any real number such that

$$
\lambda<\liminf _{r \rightarrow 0} \frac{\mu(B(x, r))}{\nu(B(x, r))} .
$$

Then there exists $\delta_{1}>0$ such that

$$
\mu(B(x, r))>\lambda \nu(B(x, r))
$$

whenever $r \in\left(0, \delta_{1}\right]$. We write

$$
\begin{aligned}
v_{\rho, \mu}(x, t)= & \int_{|x-y|>\delta_{1}} t^{-n / 2} \exp \left(-\rho \frac{|x-y|^{2}}{t}\right) \mu(d y) \\
& +\int_{|x-y|<\delta_{1}} t^{-n / 2} \exp \left(-\rho \frac{|x-y|^{2}}{t}\right) \mu(d y)=I(t)+J(t) .
\end{aligned}
$$

By (36) $I(t)=o\left(v_{\rho, v}(x, t)\right)$ as $t \rightarrow 0$. Consider $J(t)$ and put

$$
M(\gamma)=\int_{|x-y|<\gamma} \mu(d y), \quad N(\gamma)=\int_{|x-y|<\gamma} \nu(d y) .
$$

Then

$$
\int_{[0, \gamma]} d M(r)=M(\gamma), \quad \int_{[0, \gamma]} d N(\rho)=N(\gamma)
$$

and consequently $M(\gamma)>\lambda N(\gamma)$ for all $\gamma \in\left(0, \delta_{1}\right.$ ]. For all $\gamma>0$ we write

$$
V_{t}(\gamma)=t^{-n / 2} \exp \left(-\rho \frac{\gamma^{2}}{t}\right)
$$


so that

$$
J(t)=\int_{\left[0, \delta_{1}\right]} V_{t}(\gamma) d M(\gamma)
$$

Integrating by parts, we obtain

$$
J(t)=V_{t}\left(\delta_{1}\right) M\left(\delta_{i}\right)-\int_{0}^{\delta_{1}} V_{t}^{\prime}(\gamma) M(\gamma) d \gamma
$$

Therefore, since $V_{t}>0$ and $V_{t}^{\prime}<0$, we have

$$
\begin{aligned}
J(t) & >\lambda\left\{V_{t}\left(\delta_{1}\right) N\left(\delta_{1}\right)-\int_{0}^{\delta_{1}} V_{t}^{\prime}(\gamma) N(\gamma) d \gamma\right\} \\
& =\lambda \int_{\left[0, \delta_{1}\right]} V_{t}(\gamma) d N(\gamma)=\lambda \int_{|x-y|<\delta_{1}} t^{-n / 2} \exp \left(-\rho \frac{|x-y|^{2}}{t}\right) \nu(d y) .
\end{aligned}
$$

The last inequality implies

$$
\begin{aligned}
\frac{J(t)}{v_{\rho, \nu}(x, t)} & >\lambda \frac{1}{v_{\rho, \nu}(x, t)} \int_{|x-y|<\delta_{1}} t^{-n / 2} \exp \left(-\rho \frac{|x-y|^{2}}{t}\right) \nu(d y) \\
& =\lambda\left[1-\frac{1}{v_{\rho, \nu}(x, t)} \int_{|x-y|>\delta_{1}} t^{-n / 2} \exp \left(-\rho \frac{|x-y|^{2}}{t}\right) \nu(d y)\right] .
\end{aligned}
$$

Now letting $t \rightarrow 0$ and using (36) we deduce the desired result.

Throughout this section we shall assume that the system (24) is uniformly parabolic in $R_{n} \times[0, T]$ and satisfies $\left(\mathrm{A}_{1}\right),\left(\mathrm{A}_{2}\right),\left(\mathrm{A}_{3}\right)$ and $(27)$.

THEOREM 6.1. Let $\left\{u_{i}(x, t)\right\}(i=1, \ldots, N)$ be a non-negative solution of (24) given by the formulae (31), (32). If $\nu_{i}$ are positive Borel measures satisfying (34) and such that $\nu_{i}(B(x, r))>0(i=1, \ldots, N)$ for all $r \in(0, \delta]$ for some positive constant $\delta$, then

$$
\begin{aligned}
\lim \sup _{t \rightarrow 0} \frac{u_{i}(x, t)}{v_{x, v}(x, t)} & \leqslant C \sum_{j=1}^{N} \lim \sup _{r \rightarrow 0} \frac{\mu_{j}(B(x, r))}{v_{i}(B(x, r))}, \\
C_{1} \liminf _{r \rightarrow 0} \frac{\mu_{i}(B(x, r))}{v_{i}(B(x, r))} & \leqslant \liminf _{t \rightarrow 0} \frac{u_{i}(x, t)}{v_{x_{1}, v_{i}}(x, t)} \quad(i=1, \ldots, N),
\end{aligned}
$$

$C, C_{1}, \chi$ and $\chi_{1}$ are the constants appearing in the estimates (5) (with $m=1$ ) and Corollary 4.2.

Proof. The formulae (37) and (38) follow from the estimates (5), Corollary 4.2 and Lemma 6.4. 
REMARK 6.1. In the special case of Theorem 6.1 , where $\nu_{i}(i=1, \ldots, N)$ is n-dimensional Lebesgue measure, we have

$$
v_{x, y_{1}}(x, t)=\left(\frac{2 \pi}{x}\right)^{n / 2} \text { and } v_{x_{1}, y_{i}}(x, t)=\left(\frac{2 \pi}{\chi_{1}}\right)^{n / 2} .
$$

In this case Theorem 6.1 can be stated as follows

$$
K_{1}\left(\underline{D} \mu_{i}\right)(x) \leqslant \liminf _{t \rightarrow 0} u_{i}(x, t) \leqslant \limsup _{t \rightarrow 0} u_{i}(x, t) \leqslant K_{2} \sum_{j=1}^{N}\left(\bar{D} \mu_{j}\right)(x),
$$

where $K_{1}$ and $K_{2}$ are positive constants depending on $C, C_{1}, \chi, \chi_{1}$ and $n$.

Now we give some applications indicating the utility of Theorem 6.1.

Let $h$ be a positive, non-decreasing, differentiable function on $(0, \infty)$. Put $h(0)=\lim _{r \rightarrow 0} h(r)$.

If $h(0)>0$, we put $\omega_{h}(t)=t^{-n / 2} h(0)$, whilst if $h(0)=0$, we put

$$
\omega_{h, x}(t)=t^{(-n / 2)-1} \int_{0}^{\infty} h(\sqrt{s}) \exp \left(-\frac{\chi s}{t}\right) d s
$$

and

$$
\omega_{h, \chi_{1}}(t)=t^{(-n / 2)-1} \int_{0}^{\infty} h(\sqrt{s}) \exp \left(-\frac{\chi_{1} s}{t}\right) d s .
$$

We assume that the above integrals exist for all small positive $t$.

TheOREM 6.2. Let $h_{i}(i=1, \ldots, N)$ be functions as described above and let $\left\{u_{i}(x, t)\right\}(i=1, \ldots, N)$ be a non-negative solution of (24) given by (31) and (32).

If $h_{i}(0)>0$, then

$$
\begin{aligned}
C_{1} \liminf _{r \rightarrow 0} \frac{\mu_{i}(B(x, r))}{h_{i}(r)} & \leqslant \liminf _{t \rightarrow 0} \frac{u_{i}(x, t)}{\omega_{h_{h}}(t)}<\limsup \frac{u_{i}(x, t)}{\omega_{h_{1}}(t)} \\
& \leqslant C \sum_{j=1}^{N} \limsup _{r \rightarrow 0} \frac{\mu_{j}(B(x, r))}{h_{i}(r)}
\end{aligned}
$$

whilst if $h_{i}(0)=0$, then

$$
\underset{t \rightarrow 0}{\limsup } \frac{u_{i}(x, t)}{\omega_{h_{i} \chi}(x, t)} \leqslant C \chi \sum_{j=1}^{N} \limsup _{r \rightarrow 0} \frac{\mu_{j}(B(x, r))}{h_{i}(r)}
$$

and

$$
C_{1} \chi_{1} \liminf _{r \rightarrow 0} \frac{\mu_{i}(B(x, r))}{h_{i}(r)} \leqslant \liminf _{t \rightarrow 0} \frac{u_{i}(x, t)}{\omega_{h, \chi_{1}}(x, t)}
$$


Proof. We shall only consider the case $h_{i}(0)=0$. Since $h_{i}$ is differentiable on $(0, \infty)$ we have

$$
\begin{aligned}
\omega_{h, \gamma}(t) & =\frac{1}{\gamma} t^{-n / 2} \int_{0}^{\infty} \frac{d}{d s}\left(h_{i}(\sqrt{s})\right) \exp \left(-\frac{\gamma s}{t}\right) d s \\
& =\frac{1}{\gamma} t^{-n / 2} \int_{0}^{\infty} h_{i}^{\prime}(\sqrt{s}) \exp \left(-\frac{\gamma s}{t}\right) \frac{d s}{2 \sqrt{s}},
\end{aligned}
$$

where $\gamma=\chi, \chi_{1}$. Hence, if $\sigma_{n}$ denotes the surface area of the unit ball in $R_{n}$

$$
\omega_{h_{,}, \gamma}(t)=\frac{1}{\gamma} \int_{R_{n}} t^{-n / 2} \exp \left(-\frac{\gamma|x-y|^{2}}{t}\right) v_{i}(d y)
$$

where

$$
\nu_{i}(d y)=\sigma_{n}^{-1} h_{i}^{\prime}(|x-y|)|x-y|^{1-n} d y .
$$

Furthermore, for any $r>0$

$$
\nu_{i}(B(x, r))=\int_{|x-y|<r} \sigma_{n}^{-1} h_{i}^{\prime}(|x-y|)|x-y|^{1-n} d y=\int_{0}^{r} h_{i}^{\prime}(\rho) d \rho=h_{i}(r) .
$$

Applying Theorem 6.1 with $v_{h_{1},}(x, t)=\chi \omega_{h_{1}, x}(t)$ and $\nu_{h_{1}, x_{1}}(x, t)=\chi_{1} \omega_{h_{1}, x_{1}}(t)$ we obtain (40) and (41).

As an immediate consequence of Theorem 6.2 we obtain the following result on the rate of growth of $u_{i}(x, t)(i=1, \ldots, N)$ as $t \rightarrow 0$.

Corollary 6.1. Let $\left\{u_{i}(x, t)\right\}(i=1, \ldots, N)$ be a non-negative solution of (24) given by (31) and (32). Let $0<a \leqslant n$ and define

$$
v_{n-a}=\frac{\pi^{n / 2} 2^{a}}{\Gamma((n-a) / 2+1)} .
$$

Then for each $x \in R_{n}$

$$
\begin{aligned}
K_{1} \liminf _{r \rightarrow 0} \frac{\mu_{i}(B(x, r))}{v_{n-a} r^{n-a}} & \leqslant \liminf _{t \rightarrow 0} t^{a / 2} u_{i}(x, t)<\underset{t \rightarrow 0}{\lim \sup } t^{a / 2} u_{i}(x, t) \\
& \leqslant K_{1} \sum_{j=1}^{N} \limsup _{r \rightarrow 0} \frac{\mu_{j}(B(x, r))}{v_{n-a} r^{n-a}} \quad(i=1, \ldots, N),
\end{aligned}
$$

where $K_{1}, K_{2}$ are positive constants depending on $a, \chi, \chi_{1}, C$ and $C_{1}$.

PRoOF. Put $h_{i}(r)=v_{n-a} r^{n-a}$. If $a=n$, then $h_{i}(0)=v_{0}=2^{n} \pi^{n / 2}$ and $\omega_{h_{i}}(t)=$ $t^{-n / 2} v_{0}$. If $a<n$, then $h_{i}(0)=0$ and

$$
\omega_{h, \beta}(t)=t^{-n / 2} \int_{0}^{\infty} v_{n-a} s^{(n-a) / 2} \exp \left(-\beta \frac{s}{t}\right) d s=\beta^{-(n-a) / 2-1} t^{-n / 2} .
$$

Now we shall construct a non-negative solution of the form (31), (32) of the system (24), which has a prescribed maximum rate of growth as $t \rightarrow 0$. 
THEOREM 6.3. Let $\left\{h_{i}\right\}(i=1, \ldots, N)$ be positive, increasing and differentiable functions on $R_{n}$. Suppose that $h_{i}(r) \rightarrow 0$ and $r^{-n} h_{i}(r)$ as $r \rightarrow 0(i=1, \ldots, N)$. Then there exist non-zero, singular measures $\nu_{i}(i=1, \ldots, N)$ on $R_{n}$, which are positive and have compact support, such that if

$$
u_{i}(x, t)=\int_{R_{n}} \sum_{j=1}^{N} \Gamma_{i j}(x, t ; y, 0) \nu_{j}(d y) \quad(i=1, \ldots, N),
$$

then $\lim _{t \rightarrow 0} u_{i}(x, t)=\infty$, for almost every $\left[\nu_{i}\right] x \in R_{n}$, and $u_{i}(x, t)=0\left(\omega_{i}(t)\right)$ as $t \rightarrow 0(i=1, \ldots, N)$, for all $x \in R_{n}$, where $\omega_{i}=\omega_{h_{i}}$ if $h_{i}(0)>0$ and $\omega_{i}=\omega_{h_{i} x}(t)$ if $h_{i}(0)=0$.

Proof. We sketch essential ideas. Let $\mu_{h_{3}}$ denote the Hausdorff measure constructed from $h_{i}$. A theorem of Dvoretsky (1971) implies that there exists a compact set $K_{i} \subset R_{n}$ such that $0<\mu_{h_{i}}\left(K_{i}\right)<\infty$. By the lemma of Hayman and Kennedy (1976) page 223; there exists a positive measure $\nu_{i}$ on $K_{i}$ such that $0<\nu_{i}\left(K_{i}\right)<\infty$, and for each $x$ and all $r \in(0,1)$,

$$
\nu_{i}(B(x, r))<B h_{i}(r)
$$

where $B$ is a positive constant. Since $r^{n} / h_{i}(r) \rightarrow 0$ as $r \rightarrow 0$ and $\mu_{h_{i}}\left(K_{i}\right)<\infty$ $(i=1, \ldots, N)$ the $n$-dimensional Lebesgue measure of $K_{i}$ is zero (see Hayman, Kennedy (1976), p. 221). Therefore $\nu_{i}$ are singular with respect to the Lebesgue measure. Put

$$
u_{i}(x, t)=\int_{R_{n}} \sum_{j=1}^{N} \Gamma_{i j}(x, t ; y, 0) v_{j}(d y) \quad(i=1, \ldots, N),
$$

the result follows from Lemma 6.1, Remark 6.1.

In Theorem 6.4 below we estimate the size of the singularities of solution given by (31), (32) in terms of Hausdorff measure and dimension. For a bounded set in $R_{n}$, the Hausdorff dimension is defined in Hayman and Kennedy (1976) page 222. For an arbitrary set $S$, we define the Hausdorff dimension of $S$ to be $\inf \left\{\gamma ; m_{\gamma}(S)=0\right\}$, where $m_{\gamma}$ is the Hausdorff measure constructed from $h(t)=t^{\gamma}, \gamma>0$.

LemMa 6.5. Let $0 \leqslant a \leqslant n, 1 \leqslant j \leqslant N$, let $\mu$ be a positive Borel measure on $R_{n}$ satisfying (34), and let

$$
\begin{gathered}
u_{i}(x, t)=\int_{R_{n}} \Gamma_{i j}(x, t ; y, 0) \mu(d y) \quad(i=1, \ldots, N), \\
S_{a}^{i}=\left\{x \in R_{n} ; \limsup _{t \rightarrow 0} t^{a / 2} u_{i}(x, t)=\infty\right\} \quad(i=1, \ldots, N)
\end{gathered}
$$


for $0<a<n$, and

$$
T_{a}^{i}=\left\{x \in R_{n} ; \limsup _{t \rightarrow 0} t^{a / 2} u_{i}(x, t)>0\right\} \quad(i=1, \ldots, N)
$$

for $0<a<n$, then $m_{n-a}\left(S_{a}^{i}\right)=0(i=1, \ldots, N)$ and the Hausdorff dimension of $T_{a}^{i}$ does not exceed $n-a$.

Proof. We can assume that $S_{a}^{i}$ and $T_{a}^{i}$ are bounded. We shall consider only the case $0 \leqslant a<n$. By Corollary 6.1

$$
\limsup _{t \rightarrow 0} t^{a / 2} u_{i}(x, t) \leqslant K_{2} \limsup _{r \rightarrow 0} \frac{\mu(B(x, r))}{v_{n-a} r^{n-a}}
$$

for all $x \in R_{n}$. Furthermore, if $I(x, r)$ denotes the closed cube with centre $x$, edges of length $2 r$, and sides orthogonal to the coordinate axes, then

$$
\begin{aligned}
\underset{r \rightarrow 0}{\limsup } \frac{\mu(B(x, r))}{v_{n-a} r^{n-a}} & \leqslant A \underset{r \rightarrow 0}{\limsup } \frac{\mu(I(x, r))}{(2 r \sqrt{n})^{n-a}} \\
& \leqslant A \lim _{\delta \rightarrow 0}\left[\sup \left\{\frac{\mu(I)}{h(d(I))} ; x \in I, d(I)<\delta\right\}\right],
\end{aligned}
$$

where $I$ denotes any non-trivial interval, $d(I)$ is the diameter of $I$, and $h(s)=$ $s^{n-a}$. Therefore, if $Z$ denotes the set where this last upper limit is infinite, we have $S_{a}^{i} \subset Z$. Since $m_{n-a}(Z)=0$ (see Roger and Taylor (1961), Lemma 4), $m_{n-a}\left(S_{a}^{i}\right)=0$ as required. If $0<\gamma<a \leqslant n$, then $T_{a}^{i} \subset S_{\gamma}^{i}$ so that $m_{n-\gamma}\left(T_{a}^{i}\right)=$ 0 . Therefore the Hausdorff dimension of $T_{a}^{i}$ is at most inf $\{n-\gamma ; 0<\gamma<a\}=$ $n-a$.

TheOREM 6.4. Let $0 \leqslant a \leqslant n$ and $\left\{u_{i}(x, t)\right\}(i=1, \ldots, N)$ be a non-negative solution of (24) given by (31), (32). If

$$
S_{a}^{i}=\left\{x \in R_{n} ; \limsup _{t \rightarrow 0} t^{a / 2} u_{i}(x, t)=\infty\right\}
$$

for $0<a<n$, and

$$
T_{a}^{i}=\left\{x \in R_{n} ; \limsup _{t \rightarrow 0} u_{i}(x, t)>0\right\} \quad(i=1, \ldots, N)
$$

for $0 \leqslant a \leqslant n$, then $m_{n-a}\left(S_{a}^{i}\right)=0(i=1, \ldots, N)$ and the Hausdorff dimension of $T_{a}^{i}$ does not exceed $n-a$.

Proof. Put

$$
\begin{aligned}
& u_{j}^{i}(x, t)=\int_{R_{n}} \Gamma_{i j}(x, t ; y, 0) \mu_{j}(d y) \quad(i, j=1, \ldots, N), \\
& u_{i}(x, t)=u_{1}^{i}(x, t)+\cdots+u_{N}^{i}(x, t) \quad(i=1, \ldots, N) .
\end{aligned}
$$


Now define

$$
\begin{aligned}
& S_{a j}^{i}=\left\{x \in R_{n} ; \limsup _{t \rightarrow 0} t^{a / 2} u_{j}^{i}(x, t)=\infty\right\}, \\
& T_{a j}^{i}=\left\{x \in R_{n} ; \limsup _{t \rightarrow 0} t^{a / 2} u_{j}^{i}(x, t)>0\right\} \quad(i, j=1, \ldots, N) .
\end{aligned}
$$

The result follows from Lemma 6.5 and from the fact that

$$
S_{a}^{i}=\bigcup_{j=1}^{N} S_{a j}^{i}, \quad T_{a}^{i}=\bigcup_{j=1}^{N} T_{a j}^{i}
$$

Finally, we give mild conditions under which the measures $\left\{u_{j}\right\} \quad j=$ $1, \ldots, N)$ in (31) are supported by singletons, are null or are absolutely continuous with respect to the Lebesgue measure.

THEOREM 6.5. Let $\left\{u_{i}(x, t)\right\}(i=1, \ldots, N)$ be a non-negative solution of (24) given by (31), (32) and let

$$
E_{i}=\left\{x \in R_{n} ; \lim _{t \rightarrow 0} u_{i}(x, t) \text { exists }\right\} \quad(i=1, \ldots, N) .
$$

If $\lim _{t \rightarrow 0} u_{i}(x, t)=0$ almost everywhere on $E_{i}(i=1, \ldots, N)$, and $\lim _{t \rightarrow 0} u_{i}(x, t)<\infty$ on $E_{i}-\left\{x_{0}^{i}\right\}(i=1, \ldots, N)$, then

$$
u_{i}(x, t)=\sum_{j=1}^{N} \mu_{j}\left(x_{0}^{i}\right) \Gamma_{i j}\left(x, t ; x_{0}^{i}, 0\right) \quad(i=1, \ldots, N)
$$

on $R_{n} \times(0, T]$

Proof. By the Lebesgue decomposition theorem we can write $\mu_{i}=\mu_{i}^{a}+\mu_{i}^{s}$, where $\mu_{i}^{a}, \mu_{i}^{s}$ are positive measures, $\mu_{i}^{a}$ is absolutely continuous and $\mu_{i}^{s}$ is singular with respect to the Lebesgue measure. If $\left(D \mu_{i}^{s}\right)(x)=\infty$, then since $\mu_{i}^{a}>0$, we have

$$
\infty=\left(D \mu_{i}^{s}\right)(x)<\left(\underline{D} \mu_{i}\right)(x)
$$

and hence $\left(D \mu_{i}\right)(x)=\infty$. Therefore

$$
\left\{x ;\left(D \mu_{i}^{s}\right)(x)=\infty\right\} \subset\left\{x \in R_{n} ;\left(D \mu_{i}\right)(x)=\infty\right\}=F_{i}
$$

and hence by Lemma 6.1, $\mu_{i}^{s}\left(R_{n}-F_{i}\right)=0$. Further on $E_{i}-\left\{x_{0}^{i}\right\}$

$$
K_{2}\left(D \mu_{i}^{s}\right)(x) \leqslant K_{2}\left(\underline{D}_{\mu_{i}}\right)(x)<\lim _{t \rightarrow 0} u_{i}(x, t) \text {, }
$$

by Remark 6.1, and hence Lemma 6.1 implies that $\mu_{i}^{s}\left(E_{i}-\left\{x_{0}^{i}\right\}\right)=0$. By Remark 6.1 $F_{i} \subset E_{i}$, so that $\mu_{i}^{s}\left(R_{n}-E_{i}\right)=0$ and hence $\mu_{i}^{s}$ is supported by $\left\{x_{0}^{i}\right\}$. Further, $\left(D \mu_{i}\right)(x)$ exists and is finite almost everywhere and $\mu_{i}^{a}(d x)=$ $\left(D \mu_{i}\right)(x) d x$. Hence $\left(D \mu_{i}\right)(x)=\lim _{t \rightarrow 0} u_{i}(x, t)$ almost everywhere, by Theorem 
3.1, so that $\mu_{i}^{a}(d x)=\lim _{t \rightarrow 0} u_{i}(x, t) d x$, which implies that $\mu_{i}^{a}=0$. Hence $\mu_{i}$ is supported by $\left\{x_{0}^{i}\right\}$ and the result follows.

THEOREM 6.6. Let $\left\{u_{i}(x, t)\right\}(i=1, \ldots, N)$ be a non-negative solution of (24) and let

$$
E_{i}=\left\{x \in R_{n} ; \lim _{t \rightarrow 0} u_{i}(x, t) \text { exists }\right\} \quad(i=1, \ldots, N) .
$$

If $\lim _{t \rightarrow 0} u_{i}(x, t)<\infty$ everywhere on $E_{i}$ and $\lim _{t \rightarrow 0} u_{i}(x, t)=0$ almost everywhere on $E_{i}(i=1, \ldots, N)$, then $u_{i} \equiv 0$ on $R_{n} \times(0, T](i=1, \ldots, N)$.

Proof. Let $\left\{x_{0}^{i}\right\} \in E_{i}(i=1, \ldots, N)$. By Theorem 6.5 we have

$$
u_{i}(x, t)=\sum_{j=1}^{N} \mu_{j}\left(x_{0}^{j}\right) \Gamma_{i j}\left(x, t ; x_{0}^{j}, 0\right) \quad(i=1, \ldots, N)
$$

on $R_{n} \times(0, T]$. Since $\lim _{t \rightarrow 0} u_{i}\left(x_{0}^{i}, t\right)<\infty$ and $\Gamma_{i i}$ has a pole at $x=x_{0}^{i}$ we must have $\mu_{i}\left(x_{0}^{i}\right)=0(i=1, \ldots, N)$ and therefore $u_{i} \equiv 0$ on $R_{n} \times(0, T](i=$ $1, \ldots, N)$.

THEOREM 6.7. Let $\left\{u_{i}(x, t)\right\}(i=1, \ldots, N)$ be a non-negative solution of (24) given by (31), (32) such that

$$
\lim \sup u_{i}(x, t)<\infty \quad(i=1, \ldots, N)
$$

for all $x \in R_{n}$, then there exist non-negative measurable functions $f_{i}(i=$ $1, \ldots, N)$ such that $\mu_{i}(d x)=f_{i}(x) d x(i=1, \ldots, N)$.

Proof. In view of Corollary 4.2 we have

$$
\begin{aligned}
C_{1} t^{-n / 2} e^{-x_{1}} \int_{|x-y|<v_{t}} \mu_{i}(d x) & \leqslant \int_{R_{n}} \Gamma_{i i}(x, t ; y, 0) \mu_{i}(d y) \\
& <\int_{R_{n} j=1} \sum_{i j}^{N} \Gamma_{i j}(x, t ; y, 0) \mu_{j}(d y)
\end{aligned}
$$

and the result follows from Lemma 6.3.

\section{The rate of special decay of non-negative solutions of weakly coupled parabolic system}

The main aim of this section is to prove that if $\left\{u_{i}(x, t)\right\}(i=1, \ldots, N)$ is non-negative solution of the system (24) such that $u_{i}\left(x, t_{0}\right)=O\left(\exp \left(-\phi|x|^{2}\right)\right)$ for some positive constant $\phi$ depending on $t_{0}$, then $u_{i} \equiv 0(i=1, \ldots, N)$. In the sequel we assume that the system (24) satisfies $\left(A_{1}\right),\left(A_{2}\right),\left(A_{3}\right)$ and $(27)$. 
TheOREM 7.1. Let $\left\{u_{i}(x, t)\right\}(i=1, \ldots, N)$ be a non-negative solution of the system (24) and

$$
M_{k}(r)=\max _{|x|=r} u_{k}\left(x, t_{0}\right) \quad(k=1, \ldots, N) .
$$

If, for some index $j$,

$$
\liminf _{r \rightarrow \infty}\left\{r^{-1} \log M_{j}(r)+\chi_{1} \frac{r}{t_{0}}\right\} \leqslant 0,
$$

then the measure $\mu_{j}$ is concentrated at 0 . ( $\mu_{j}$ denotes the measure appearing in the representation formulae (31), (32).)

Proof. Suppose that $\mu_{\mathrm{j}}$ is not concentrated at 0 , then there is a closed ball $B \subset R_{n}$, with centre $a$ and radius $\rho$, such that $0 \notin B$ (so that $|a|>\rho$ ) and $\mu_{j}(B)>0$. Then it follows from (31) that

$$
\begin{aligned}
C_{1} t_{0}^{-n / 2} \int_{B} \exp \left(-\chi_{1} \frac{|x-y|^{2}}{t_{0}}\right) \mu_{j}(d y) & \leqslant C_{1} t_{0}^{-n / 2} \int_{R_{n}} \exp \left(-\chi_{1} \frac{|x-y|^{2}}{t_{0}}\right) \mu_{j}(d y) \\
& \leqslant \mu_{j}\left(x, t_{0}\right) .
\end{aligned}
$$

Since $|x-y| \leqslant|x-a|+\rho$, for all $y \in B$,

$$
\begin{aligned}
& \mu_{j}(B) C_{1} t_{0}^{-n / 2} \exp \left(-\frac{\chi_{1}}{t_{0}}(|x-a|+\rho)^{2}\right) \\
& \leqslant C_{1} t_{0}^{-n / 2} \int_{B} \exp \left(-\frac{\chi_{1}}{t_{0}}(|x-a|+\rho)^{2}\right) \mu_{j}(d y) \leqslant u_{j}\left(x, t_{0}\right) .
\end{aligned}
$$

It is easy to see that

$$
\min \{|x-a|+\rho ;|x|=r\}=r-(|a|-\rho)
$$

and hence

$$
\mu_{j}(B) C_{1} t_{0}^{-n / 2} \exp \left\{-\frac{\chi_{1}}{t_{0}}[r-(|a|-\rho)]^{2}\right\} \leqslant M_{j}(r)
$$

Therefore, wherever $r \geqslant|a|+\rho$

$$
r^{-1} \log \left(\mu_{j}(B) C_{1} t_{0}^{-n / 2}\right)-\frac{\chi_{1}}{t_{0}}\left\{r+\frac{(|a|-\rho)^{2}}{r}-2(|a|-\rho)\right\}<r^{-1} \log M_{j}(r)
$$

and hence

$$
2 \frac{\chi_{1}}{t_{0}}(|a|-\rho)<\liminf _{r \rightarrow \infty}\left(r^{-1} \log M_{j}(r)+\frac{\chi_{1} r}{t_{0}}\right) .
$$

The result now follows at once. 
COROLlary 7.1. If $\left\{u_{i}(x, t)\right\}(i=1, \ldots, N)$ is a non-negative solution of the system (24) such that

$$
\liminf _{r \rightarrow \infty}\left\{r^{-1} \log M_{j}(r)+\chi_{1} \frac{r}{t_{0}}\right\}<0 \quad(j=1, \ldots, N),
$$

then there exist constants $A_{k}(k=1, \ldots, N)$ such that

$$
u_{i}(x, t)=\sum_{j=1}^{N} A_{j} \Gamma_{i j}(x, t ; 0,0)
$$

for all $(x, t) \in R_{n} \times\left(0, t_{0}\right]$.

THEOREM 7.2. If $\left\{u_{i}(x, t)\right\}(i=1, \ldots, N)$ is a non-negative solution of the system (24) in $R_{n} \times\left(0, t_{0}\right]$ and

$$
u_{i}\left(x, t_{0}\right) \leqslant \lambda \exp \left(-\alpha|x|^{2}\right) \quad(i=1, \ldots, N)
$$

for $x \in R_{n}$, for some positive constant $\alpha>\chi_{1} / t_{0}$ and $\lambda>0$, then $u_{i} \equiv 0(i=$ $1, \ldots, N)$.

Proof. In this situation, we have

$$
\frac{\log M_{j}(r)}{r}+\chi_{1} \frac{r}{t_{0}} \leqslant o(1)-\alpha r+\frac{\chi_{1} r}{t_{0}} \rightarrow-\infty
$$

as $r \rightarrow \infty$. It follows from Corollary 7.1 that

$$
u_{i}(x, t)=\sum_{j=1}^{N} A_{j} \Gamma_{i j}(x, t ; 0,0)
$$

for some positive constants $A_{j}$. By Corollary 4.2

$$
A_{k} \leqslant \frac{u_{k}\left(x, t_{0}\right)}{\Gamma_{k k}\left(x, t_{0} ; 0,0\right)} \leqslant \frac{u_{k}\left(x, t_{0}\right)}{C_{1} t_{0}^{-n / 2} \exp \left(-\chi_{1} \frac{|x|^{2}}{t_{0}}\right)}<\frac{\lambda \exp \left(-\alpha|x|^{2}\right)}{C_{1} t_{0}^{-n / 2} \exp \left(-\chi_{1} \frac{|x|^{2}}{t_{0}}\right)} \rightarrow 0
$$

as $|x| \rightarrow \infty$, from which it follows that $A_{j}=0(j=1, \ldots, N)$, and therefore $u_{i} \equiv 0(i=1, \ldots, N)$.

\section{Local Fatou property}

Consider the weakly coupled parabolic system (24) whose the coefficients are independent of $t$, that is,

$$
\begin{aligned}
L_{i}\left(u_{1}, \ldots, u_{N}\right)=\sum_{|k|=1,2} A_{k}^{i i}(x) D_{x}^{k} u_{i}+\sum_{j=0}^{N} A_{0}^{i j}(x) u_{j}-\frac{\partial u_{i}}{\partial t} & =0 \\
& (i=1, \ldots, N) .
\end{aligned}
$$

We shall prove a local version of Theorem 3.1. 
Throughout this section we will assume that the coefficients satisfy the following conditions:

(I) The coefficients $A_{k}^{i i}(x)(|k|=1,2, i=1, \ldots, N), A_{0}^{i j}(x)(i, j=1, \ldots, N)$, and the derivatives $D^{h} A_{k}^{i i}(x)(|k|=1,2,|h|<|k|, i=1, \ldots, N)$ are Hölder continuous on compact subsets of $R_{n}$.

(II) For each $i$ the matrix $\left\{A_{k}^{i j}(x)\right\}(|k|=2)$ is symmetric and positive definite for any $x \in R_{n}$.

(III) $A_{0}^{i j}(x)>0$ in $R_{n}, i \neq j$.

It follows from (II) that each operator $L_{i}$ is uniformly parabolic in every cylinder $B(0, a) \times[0, T]$. It is clear that for every $a>0$ there exists an operator $L_{a}=\left(L_{1}^{a}, \ldots, L_{N}^{a}\right)$, where

$$
L_{i}^{a}\left(v_{i}, \ldots, v_{N}\right)=\sum_{|k|=1,2} A_{k a}^{i i}(x) D^{k} v_{i}+\sum_{j=1}^{N} A_{0 a}^{i j}(x) v_{j}-\frac{\partial v_{i}}{\partial t}
$$

satisfy the following conditions

(Ia) The coefficients $A_{k a}^{i i}, A_{0 a}^{i j}(|k|=1,2, i, j=1, \ldots, N)$ and the derivatives $D^{h} A_{k a}^{i i}(|h|<|k|,|k|=1,2, i=1, \ldots, N)$ are bounded and Hölder continuous in $R_{n}$.

(IIa) There exists a positive constant $\delta(a)$ such that

$$
\sum_{|k|=2} A_{k a}^{i i}(x) \xi^{k}>\delta(a)|\xi|^{2} \quad(i=1, \ldots, N)
$$

for all $x \in R_{n}$ and $\xi \in R_{n}$.

$$
\begin{aligned}
& \text { (IIIa) } A_{o a}^{i j}(x)>0 \text { in } R_{n}, i \neq j . \\
& \text { (IVa) } A_{k}^{i i}(x)=A_{k a}^{i i}(x) \text {, and }
\end{aligned}
$$

$$
A_{0}^{i j}(x)=A_{0 a}^{i j}(x)
$$

$(i, j=1, \ldots, N,|k|=1,2)$, for all $|x|<a$.

Under the assumptions (Ia), (IIa) and (IIIa) the fundamental matrix $\left\{\Gamma_{i j}^{a}(x, t ; y, r)\right\} \quad(i, j=1, \ldots, N)$ for the system $L_{i}^{a}\left(u_{i}, \ldots, u_{N}\right)=0 \quad(i=$ $1, \ldots, N)$ exists. By Corollary 4.2 all the functions $\Gamma_{i j}^{a}$ are nonnegative and

$$
C_{i}(a)(t-\tau)^{-n / 2} \exp \left(-\chi_{1}(a) \frac{|x-y|^{2}}{t-\tau}\right)<\Gamma_{i i}^{a}(x, t ; y, \tau)
$$

$$
(i=1, \ldots, N)
$$

for $(x, t),(y, \tau) \in R_{n} \times[0, T], 0<\tau<t<T$.

As in Section 3 we introduce the truncated parabolic region

$$
\Gamma_{\gamma}^{h}\left(x_{0}\right)=\left\{(x, t) ; 0<\left|x-x_{0}\right|<\gamma \sqrt{t}, 0<t<h\right\} .
$$


We shall say that the function $u(x, t)$ defined in $R_{n} \times(0, h]$ is parabolically bounded at $x_{0}$ if for some $\beta>0$

$$
\sup _{(x, t) \in \Gamma_{\beta}^{\prime}\left(x_{0}\right)}|u(x, t)|<\infty .
$$

Notice that the parabolic boundedness at $x_{0}$ requires a condition on only one truncated parabolic region whose vertex is at $x_{0}$, while the existence of the parabolic limit (see Section 3 ) at $x_{0}$ requires a condition on all parabolic regions at $x_{0}$.

LEMMA 8.1. Let $u(x, t)$ be a continuous function in $R_{n} \times(0, T]$ parabolically bounded at each point of a measurable set $E$, then for every $\varepsilon>0$ there is a compact set $E_{1} \subset E$ such that

(1) $m\left(E-E_{1}\right)<\varepsilon$,

(2) for any $\alpha>0$, there is a positive number $M=M(\alpha, \varepsilon)$ such that $|u(x, t)|<$ $M$ for all $(x, t) \in \Gamma_{\alpha}^{T}\left(x_{0}\right), x_{0} \in E_{1}$.

The proof is a simple modification of that of the lemma on page 201 in Stein (1970).

THEOREM 8.1. Suppose (I), (II) and (III) hold. If $\left\{u_{i}(x, t)\right\}(i=1, \ldots, N)$ is a solution of the system (42) in $R_{n} \times(0, T]$, parabolically bounded at each point $x$ of a measurable set $E \subset R_{n}$, then for almost all $x \in E$ the limits, $\mathrm{p}-\lim _{(y, t) \rightarrow(x, 0)}$ $u_{i}(y, t)$ exist.

Proof. Fix $\alpha>0$ and define the set

$$
R=\bigcup_{x_{0} \in E} \Gamma_{\alpha}^{T}\left(x_{0}\right)
$$

It follows from Lemma 8.1 that it suffices to prove the theorem when $E$ is a compact set and $\left|u_{i}(x, t)\right| \leqslant 1$ for $(x, t) \in R(i=1, \ldots, N)$. Clearly it suffices to prove that the limits, $\lim u_{i}(x, t)_{(x, t) \rightarrow\left(x_{0}, 0\right),(x, t) \in R}(i=1, \ldots, N)$ all $x_{0} \in E$. For any $m>0$ (sufficiently large) define

$$
\phi_{i m}(x)= \begin{cases}u_{i}\left(x, \frac{1}{m}\right) & \text { for }\left(x, \frac{1}{m}\right) \in R, \\ 0 & \text { elsewhere. }\end{cases}
$$

Choose $a>0$ such that

$$
R \subset B(0, a) \times(0, T]
$$

Set

$$
\phi_{i m}(x, t)=\int_{R_{n} j=1}^{N} \Gamma_{i j}^{a}(x, t ; y, 0) \phi_{j m}(y) d y
$$


and

$$
u_{i}\left(x, t+\frac{1}{m}\right)=\phi_{i m}(x, t)+\Psi_{i m}(x, t) \quad(i=1, \ldots, N) .
$$

The functions $\left\{\phi_{i m}(x)\right\}$ are uniformly bounded in $L^{\infty}\left(R_{n}\right)$ norm, and thus we can extract a subsequence which converges weakly to a function $\phi_{i}(x)$. Clearly $\left|\phi_{i}(x)\right|<1$ a.e. in $R_{n}$. For simplicity we again denote this subsequence by $\left\{\phi_{i m}(x)\right\}$.

Introducing

$$
\phi_{i}(x, t)=\int_{R_{n} j=1} \sum_{i j}^{N} \Gamma_{i j}^{a}(x, t ; y, 0) \phi_{j}(y) d y \quad(i=1, \ldots, N)
$$

and letting $m \rightarrow \infty$ in (44) one easily verifies that $\lim _{m \rightarrow \infty} \Psi_{i m}(x, t)(i=$ $1, \ldots, N)$ exists and hence

$$
u_{i}(x, t)=\phi_{i}(x, t)+\Psi_{i}(x, t) \quad(i=1, \ldots, N) .
$$

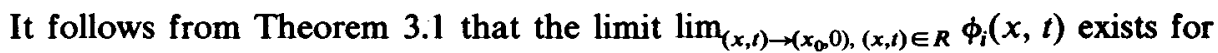
almost all $x_{0} \in E(i=1, \ldots, N)$.

To prove the existence of the limits of $\Psi_{i}$ we shall construct functions $\left\{H_{i}(x, t)\right\}$ $(i=1, \ldots, N)$ satisfying the following conditions

1) $L_{i}^{a}\left(H_{1}, \ldots, H_{N}\right)=0$ in $R_{n} \times(0, T]$,

2) $H_{i}(x, t) \geqslant 0$ for $(x, t) \in R_{n} \times(0, T](i=1, \ldots, N)$,

3) $\left|\Psi_{i}(x, t)\right| \leqslant H_{i}(x, t)$ for $(x, t) \in R(i=1, \ldots, N)$,

4) $\mathrm{p}-\lim _{(x, t) \rightarrow\left(x_{0}, 0\right)} H_{i}(x, t)=0$ for almost all $x_{0} \in E(i=1, \ldots, N)$.

We construct $H_{i}(x, t)$ in the form

$$
H_{i}(x, t)=K \int_{R_{n} j=1}^{N} \Gamma_{i j}^{a}(x, t ; y, 0) X(y) d y \quad(i=1, \ldots, N),(i=1, \ldots, N),
$$

where $X(y)$ denotes the characteristic function of the complement of $E$ and the positive constant $K$ will be chosen later. The properties 1) and 2) are obvious. Let $\partial R$ be a part of the boundary of $R$ defined by

$$
\partial R=E \times\{0\} \cup\{(x, t) ;(x, t) \in \bar{R}-R, 0<t<T\} .
$$

Note that if $(x, t) \in \partial R-E \times\{0\}$, then $\{y ;|y-x|<\alpha \sqrt{t}\} \subset R_{n}-E$. Hence it follows from (43) that

$$
\begin{aligned}
H_{i}(x, t) & =K \int_{R_{n} j=1} \sum_{i j}^{N} \Gamma_{i j}(x, t ; y, 0) X(y) d y \\
& >C_{1}(a) K \int_{R_{n}-E} t^{-n / 2} \exp \left(-\chi_{1}(a) \frac{|x-y|^{2}}{t}\right) d y \\
& >C_{1}(a) K \int_{|z|<\alpha} \exp \left(-\chi_{1}(a)|z|^{2}\right) d z .
\end{aligned}
$$


It is clear that

$$
\left|\Psi_{i}(x, t)\right| \leqslant H_{i}(x, t) \quad(i=1, \ldots, N)
$$

for $(x, t) \in \partial R-E \times\{0\}$ provided $K$ is sufficiently large. To establish 3) we first prove that

$$
\Psi_{i m}(x, t) \leqslant H_{i}(x, t) \quad(i=1, \ldots, N)
$$

for $(x, t) \in R$, for every $m$ (sufficiently large). If this were not so there would exist an $\varepsilon>0$ and an index $j$ such that

$$
\sup _{\bar{R}}\left[\Psi_{j m}(x, t)-H_{i}(x, t)\right]=\max _{i} \sup _{\bar{R}}\left[\Psi_{i m}(x, t)-H_{i}(x, t)\right]>\varepsilon .
$$

The maximum cannot be attained on $\partial R-E \times\{0\}$. Applying the maximum principle (see Szarski (1975)) we can conclude that there exists a sequence of points $\left(x^{k}, t^{k}\right)$ converging to $\left(x_{0}, 0\right) \in E \times\{0\}$ such that

$$
\Psi_{j m}\left(x^{k}, t^{k}\right)>H_{j}\left(x^{k}, t^{k}\right)+\varepsilon .
$$

On the other hand $\Phi_{j m}(x)$ is equal to $u_{j}(x, 1 / m)$ on an open set containing $E$, therefore

$$
\lim _{k \rightarrow \infty}\left[u_{j}\left(x^{k}, t^{k}+\frac{1}{m}\right)-\phi_{j m}\left(x^{k}, t^{k}\right)\right]=\lim _{k \rightarrow \infty} \Psi_{j m}\left(x^{k}, t^{k}\right)=0,
$$

this contradicts to (45). Analogously we prove $\Psi_{i m}(x, t)>-H_{i}(x, t)$ in $R$, for any $m(i=1, \ldots, m)$.

Let $E$ be a compact set in $R_{n}$. Choose $a>0$ such that $E \subset B(0, a)$.

THEOREM 8.2. Suppose the assumptions (I), (II) and (III) hold. Let $\left\{u_{i}(x, t)\right\}$ $(i=1, \ldots, N)$ be a solution of the system $(42)$ in $R_{n} \times(0, T]$ parabolically bounded at each point $x$ of a compact set $E \subset R_{n}$. Then there exists a unique decomposition

$$
u_{i}(x, t)=\int_{R_{n}} \sum_{j=1}^{N} \Gamma_{i j}^{a}(x, t ; y, 0) f_{j}(y) d y+\Psi_{i}(x, t) \quad(i=1, \ldots, N),
$$

where $f_{i} \in L^{\infty}\left(R_{n}\right)$, supp $f_{i} \subset E$ and $\mathrm{p}-\lim _{(x, t) \rightarrow\left(x_{0}, 0\right)} \Psi_{i}(x, t)=0$ for almost $x_{0} \in$ $E(i=1, \ldots, N)$.

Proof. From the proof of Theorem 8.1 it follows that such a decomposition exists. To prove the uniqueness suppose there is a second decomposition

$$
u_{i}(x, t)=\int_{R_{n}} \sum_{j=1}^{N} \Gamma_{i j}^{a}(x, t ; y, 0) \tilde{f}_{j}(y) d y+\tilde{\Psi}_{i}(x, t) \quad(i=1, \ldots, N)
$$


with properties $\tilde{f}_{i} \in L^{\infty}\left(R_{n}\right)$, supp $\tilde{f}_{i} \subset E, \quad \mathrm{p}-\lim _{(x, t) \rightarrow\left(x_{0}, 0\right)} \tilde{\Psi}_{i}(x, t)=0 \quad(i=$ $1, \ldots, N)$ for almost all $x_{0} \in E$. The relation

$$
\underset{(x, t) \rightarrow\left(x_{0}, 0\right)}{\mathrm{p}-\lim } \Psi_{i}(x, t)=\operatorname{p}_{(x, t) \rightarrow\left(x_{0}, 0\right)} \tilde{\Psi}_{i}(x, t)=0 \quad(i=1, \ldots, N)
$$

for almost all $x_{0} \in E$, implies

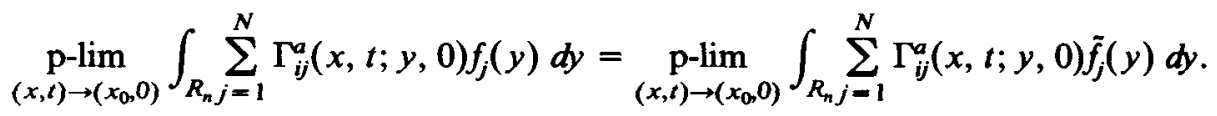

for almost all $x_{0} \in E$. From Theorem 3.1 and the fact that supp $f_{i}$, supp $\tilde{f}_{i} \subset E$ it follows that $f_{i}=\tilde{f}_{i}$ almost everywhere in $R_{n}$ and consequently $\Psi_{i}=\tilde{\Psi}_{i}$ in $R_{n} \times(0, T]$.

\section{Initial distribution problem}

To motivate the results of this section (Theorems 9.4 and 9.5) we now consider the heat equation

$$
D_{x}^{2} u-D_{t} u=0
$$

for $(x, t) \in(-\infty, \infty) \times(0, T)$. We can interpret $u(x, t)$ physically as representing the temperature in an infinite rod. Moreover, $\int_{a}^{b} u(x, t) d x, a<b$, is the quantity of heat at time $t$ in the portion $(a, b)$ of the rod. Recently Wilcox (1980) proposed the problem of constructing a non-negative solution of the heat equation (46) whose initial heat distribution exists and is equal to a given Borel measure $\mu$ on $(-\infty, \infty)$.

More precisely a non-negative solution $u$ of (46) is said to have a non-negative Borel measure $\mu$ on $(-\infty, \infty)$ as its initial heat distribution if

$$
\lim _{t \rightarrow 0} \int_{a}^{b} u(x, t) d x=\mu(a, b)
$$

for all $a<b$ such that $\mu\{a\}=\mu\{b\}=0$.

C. H. Wilcox gave a complete solution to the initial distribution problem.

THEOREM 9.1. There exists a non-negative solution $u$ of (46) whose initial heat distribution is the non-negative Borel measure $\mu$ if and only if

$$
\int_{-\infty}^{\infty} \exp \left(-\frac{x^{2}}{4 t}\right) \mu(d x)<\infty
$$

for all $t \in(0, T)$. If $\mu$ satisfies (47), then $u$ is given by

$$
u(x, t)=(4 \pi t)^{-1 / 2} \int_{-\infty}^{\infty} \exp \left(-\frac{(x-y)^{2}}{4 t}\right) \mu(d y)
$$

for all $(x, t) \in(-\infty, \infty) \times(0, T)$ and is unique. 
The above theorem is a consequence of Widder's Inversion Theorem:

If a non-negative solution $u$ of (46) has the representation (48) then for all real $a$ and $b$ with $a<b$

$$
\lim _{t \rightarrow 0} \int_{a}^{b} u(x, t) d x=\mu(a, b)+\frac{1}{2} \mu(a)+\frac{1}{2} \mu(b) .
$$

D. G. Aronson has extended Widder's Inversion Theorem and Wilcox's result to the heat equation in $R_{n} \times(0, T)$

$$
\Delta_{x} u-D_{t} u=0
$$

Let $B$ denote $\sigma$-field of Borel subset of $R_{n}$. For each $A \in B$ define

$$
\gamma_{A}(x, t)=\int_{A}(4 \pi t)^{-n / 2} \exp \left(-\frac{|x-y|^{2}}{4 t}\right) d y .
$$

Since

$$
\int_{R_{n}}(4 \pi t)^{-n / 2} \exp \left(-\frac{|x-y|^{2}}{4 t}\right) d y=1
$$

it follows that

$$
0 \leqslant \gamma_{A}(x, t)<1
$$

for $(x, t) \in R_{n} \times(0, T)$. Therefore

$$
\gamma_{A}^{+}(x)=\lim _{t \rightarrow 0} \sup \gamma_{A}(x, t) \text { and } \gamma_{A}^{-}(x)=\lim _{t \rightarrow 0} \inf \gamma_{A}(x, t)
$$

both exist in $[0,1]$ for all $x \in R_{n}$.

Let

$$
A^{\#}=\left\{x \in R_{n} ; \gamma_{A}^{+}(x) \neq \gamma_{A}^{-}(x)\right\}
$$

and define $\gamma_{A}: R_{n}-A^{\#} \rightarrow[0,1]$ by $\gamma_{A}(x)=\gamma_{A}^{ \pm}(x)$. It is obvious that

$$
\gamma_{A}^{ \pm}(x)= \begin{cases}1 & \text { for } x \in \operatorname{Int} A, \\ 0 & \text { for } x \in R_{n}-\bar{A} .\end{cases}
$$

Consequently $A^{\#} \subset \partial A \equiv \bar{A}-$ Int $A$. D. G. Aronson gave an example of $a$ bounded, open set $A \subset R_{n}$ for $n \geqslant 2$ such that $A^{\#} \neq \varnothing$.

By Widder's representation theorem (see Theorem 5.1) if $u$ is a non-negative solution of (49) then there exists a unique non-negative Borel measure $\rho$ on $R_{n}$ such that

$$
u(x, t)=(4 \pi t)^{-n / 2} \int_{R_{n}} \exp \left(-\frac{|x-y|^{2}}{4 t}\right) \rho(d y) .
$$

On the other hand, it follows from the proof of Theorem 5.1 that

$$
\int_{R_{n}} u(x, t) \exp \left(-\frac{|x|^{2}}{T}\right) d x \leqslant(2 \pi T)^{n / 2} u\left(0, \frac{T}{2}\right)
$$

for all $(x, t) \in R_{n} \times(0, T / 2)$. 
The following Theorem (due to Aronson (1981)) is a generalization of Widder's Inversion Theorem.

THEOREM 9.2. Let $u$ be a non-negative solution of (49) given by (50). If $A \in B$ is bounded and $\rho\left(A^{\#}\right)=0$, then

$$
\lim _{t \rightarrow 0} \int_{A} u(x, t) d x=\rho(\operatorname{Int} A)+\int_{\partial A} \gamma_{A}(\xi) \rho(d \xi) .
$$

SKetch of THE Proof. Using the explicit formula (50) one can show that there exists a constant $K>0$ such that

$$
0<\gamma_{A}(x, t)<K e^{-|x|^{2} /(4 T)}
$$

for all $(x, t) \in R_{n} \times(0, T / 2)$. By Fubini's Theorem we have

$$
\int_{A} u(x, t) d x=\int_{R_{n}} \gamma_{A}(x, t) \rho(d x)
$$

Therefore by the Dominated Convergence Theorem

$$
\lim _{t \rightarrow 0} \int_{A} u(x, t) d x=\int_{R_{n}} \gamma_{A}(x) \rho(d x)
$$

and (52) follows from $R_{n}=\operatorname{Int} A \cup \partial A \cup\left(R_{n}-\bar{A}\right)$.

For a set $Q \subset(-\infty, \infty)$ we define

$$
I_{Q}=\left\{(a, b] ; a_{i}, b_{i} \in Q, i=1, \ldots, n\right\},
$$

where $(a, b]=\left\{x \in R_{n} ; a_{i}<x_{i} \leqslant b_{i}, i=1, \ldots, n\right\}$.

Let $u$ be a non-negative solution of (49) on $R_{n} \times(0, T)$ and let $\mu$ be a non-negative Borel measure on $B$. The function $u$ will be said to have initial distribution $\mu$ if there exists a countable dense subset $Q \subset(-\infty, \infty)$ such that

$$
\lim _{t \rightarrow 0} \int_{(a, b]} u(x, t) d x=\mu(a, b) \text { and } \mu(\partial(a, b])=0
$$

for all $(a, b] \in I_{Q}$.

It follows from the estimation (51) that if $\mu$ is an initial distribution of $u$ then it must be $\sigma$-finite.

The following theorem is due to Aronson (1981).

THEOREM 9.3. Let $u$ be a non-negative solution of (49) in $R_{n} \times(0, T)$ and let $\mu$ be a non-negative Borel measure on $B$. Then $u$ has initial distribution $\mu$ if and only if

$$
u(x, t)=(4 \pi t)^{-n / 2} \int_{R_{n}} \exp \left(-\frac{|x-y|^{2}}{4 t}\right) \mu(d y)
$$

for $(x, t) \in R_{n} \times(0, T)$. 
SKetch of The Proof. Suppose that $u$ is given by (53) and define the Borel measure

$$
\nu(A)=\int_{A} e^{-|x|^{2} /(4 t)} \mu(d x)
$$

for $A \in B$.

Since $\nu\left(R_{n}\right)<\infty$ one can prove that there exists a countable set $E \subset$ $(-\infty, \infty)$ such that

$$
\nu\left(H_{t}^{\prime}\right)=\int_{H_{t}^{\prime}} e^{-|x|^{2} /(4 T)} \mu(d x)=0
$$

for $l=1, \ldots, n, t \in(-\infty, \infty)-E$, where

$$
H_{t}^{l}=\left\{x \in R_{n} ; x_{l}=t\right\} .
$$

In particular, it follows that

$$
\mu(S)=0
$$

for each bounded, measurable subset $S$ of any hyperplane $H_{t}^{l}, l=1, \ldots, n$, $t \in(-\infty, \infty)-E$. Since $E$ is countable, there is a countable dense set $Q \subset$ $(-\infty, \infty)-E$. If $(a, b] \in I_{Q}$ then $\partial(a, b]$ is a finite union of bounded subsets of hyperplanes $H_{t}^{l}$ for $l=1, \ldots, n$ and $t \in Q$. Consequently it follows from (54) that $\mu(\partial(a, b])=0$. On the other hand, it is easy to show that $(a, b]^{\#}=\varnothing$ and by Theorem 9.2

$$
\lim _{t \rightarrow 0} \int_{(a, b]} u(x, t) d x=\mu(a, b)
$$

for all $(a, b] \in I_{Q}$. Therefore $\mu$ is the initial distribution of $u$.

Conversely, suppose that $\mu$ is initial distribution of $u$. By Widder's representation theorem $u$ must be of the form (50), where $\rho$ is non-negative Borel measure. It is clear that $\rho$ is $\sigma$-finite. By Theorem 9.2

$$
\lim _{t \rightarrow 0} \int_{(a, b]} u(x, t) d x=\rho(a, b)+\int_{\partial(a, b]} \gamma_{A}(x) \rho(d x)
$$

for all $(a, b] \in I_{Q}$. Since $\mu$ is the initial distribution of $u$ one can prove, using the above formula, that

$$
\rho(a, b)=\mu(a, b)
$$

for all $(a, b] \in I_{Q}$. The family of sets $\left\{I_{Q}, \varnothing\right\}$ is a $\pi$-system, which generates $B$ and therefore $\mu \equiv \rho$ (see Billingsley (1979), Theorem 10.3, p. 135).

Theorems 9.2 and 9.3 can be extended to non-negative solutions of the weakly coupled parabolic systems defined by (24) satisfying assumptions $\left(\mathrm{A}_{1}\right),\left(\mathrm{A}_{2}\right),\left(\mathrm{A}_{3}\right)$ and (27). For $A \in B$ define

$$
\gamma_{A, i}(x, t)=\int_{A} \Gamma_{i i}(x, t ; y, 0) d y \quad(i=1, \ldots, N) .
$$


It follows from (5) that the $\gamma_{A, i}$ are bounded on $R_{n} \times(0, T)$. Thus

$$
\gamma_{A, i}^{+}(x)=\lim _{t \rightarrow 0} \sup \gamma_{A, i}(x, t) \text { and } \gamma_{A, i}^{-}(x, t)=\lim _{t \rightarrow 0} \inf \gamma_{A, i}(x, t)
$$

are well defined on $R_{n}$. Set

$$
A_{i}^{\#}=\left\{x \in R_{n} ; \gamma_{A, i}^{+}(x) \neq \gamma_{A, i}^{-}(x)\right\}
$$

and define $\gamma_{A, i}(x)=\gamma_{A, i}^{ \pm}(x)$ for $x \in R_{n}-A_{i}^{\#}, i=1, \ldots, N$. Using (4) and (5) it is easy to show that

$$
\lim _{t \rightarrow 0} \int_{A} \Gamma_{i j}(x, t ; y, 0) d y=0
$$

for $x \in R_{n}, A \in B$ and $i \neq j$. Moreover

$$
\gamma_{A, i}(x)= \begin{cases}1 & \text { for } x \in \operatorname{Int} A, \\ 0 & \text { for } x \in R_{n}-\bar{A} .\end{cases}
$$

The proof of the next theorem is essentially the same as the proof of Theorem 9.2.

THEOREM 9.4. Let $\left\{u_{i}(x, t)\right\}(i=1, \ldots, N)$ be a non-negative solution of (24) in $R_{n} \times(0, T)$ given by the formulae (31) and (32). If $A \in B$ is bounded and $\mu_{i}\left(A_{i}^{\#}\right)=0(i=1, \ldots, N)$ then

$$
\lim _{t \rightarrow 0} \int_{A} u_{i}(x, t) d x=\mu_{i}(\operatorname{Int} A)+\int_{\partial A} \gamma_{A, i}(x) \mu_{i}(d x), \quad i=1, \ldots, N .
$$

Let $\left\{u_{i}\right\}(i=1, \ldots, N)$ be a non-negative solution of $(24)$ in $R_{n} \times(0, T)$ and let $\left\{\mu_{i}\right\}(i=1, \ldots, N)$ be non-negative Borel measures on $B$. The solution $\left\{u_{i}\right\}$ will be said to have initial distribution $\left\{\mu_{i}\right\}$ if there exist countable dense subsets $Q_{i} \subset(-\infty, \infty)(i=1, \ldots, N)$ such that

$$
\lim _{t \rightarrow 0} \int_{(a, b]} u_{i}(x, t) d x=\mu_{i}(a, b)
$$

and $\mu_{i}(\partial(a, b])=0$ for all $(a, b] \in I_{Q}(i=1, \ldots, N)$.

THEOREM 9.5. A non-negative solution $\left\{u_{i}\right\}(i=1, \ldots, N)$ of (24) in $R_{n} \times$ $(0, T)$ has initial distribution $\left\{\mu_{i}\right\}(i=1, \ldots, N)$ if and only if

$$
u_{i}(x, t)=\int_{R_{n}} \sum_{j=1}^{N} \Gamma_{i j}(x, t ; y, 0) \mu_{j}(d y) \quad(i=1, \ldots, N)
$$

for all $(x, t) \in R_{n} \times(0, T)$. 
This result follows from Theorems 9.4 and 5.1 in the same way that Theorem 9.3 follows from Theorem 9.2 and Widder's representation theorem; the details of the proof are therefore omitted.

\section{References}

D. G. Aronson (1968), 'Non-negative solutions of linear parabolic equations', Ann. Scuola Norm. Sup. Pisa Sci. Fis. Mat. 22, 607-694.

D. G. Aronson, 'Widder's Inversion Theorem and the initial distribution problem', Siam J. Math. Anal., to appear.

P. Besala (1972), "Function classes pertaining to differential inequalities of parabolic type in unbounded regions', Ann. Polon. Math. 25, 281-291.

B. Billingsley (1979), Probability and measure (John Wiley, New Yórk).

J. Chabrowski (1967a), 'Bemerkungen über Zeichen der Elemente der Matrix der Grundlösungen für parabolishe Systeme von partiellen Differentialgleichungen zweiter Ordnung', Ann. Polon. Math. 19, 287-300.

J. Chabrowski (1967b), 'Les solutions non-négatives d'un système parabolique d'équations', Ann. Polon. Math. 19, 193-197.

J. Chabrowski (1970), 'Les propriétés des solutions non négatives d'un système parabolique d'équations', Ann. Polon. Math. 22, 223-231.

J. Chabrowski (1971), 'Certaines propriétés des solutions non négatives d'un système parabolique d'équations', Ann. Polon. Math. 24, $137-143$.

J. Chabrowski (1974), 'Representation theorems and Fatou theorems for parabolic systems in the sense of Pietrowskii', Colloq. Math. 31, 301-314.

J. Chabrowski and N. A. Watson (1980), 'Properties of solution of weakly coupled parabolic systems', to appear.

A. Dvoretzky (1971), 'A note on Hausdorff dimension functions', Proc. London Math. Soc. (3) 33, $385-451$.

S. D. Eidel'man (1969), Parabolic systems (North Holland, Amsterdam).

A. Friedman (1964), Partial differential equations of parabolic type (Prentice-Hall, Englewood Cliffs).

W. K. Hayman and P. B. Kennedy (1976), Subharmonic functions I (Academic Press, London).

R. Johnson (1971), 'Representation theorems and Fatou theorems for second-order linear parabolic differential equations', Proc. London Math. Soc. 23, 325-347.

V. A. Kondrat'ev and S. D. Eidel'man (1974), 'Positive solutions of linear partial differential equations', Trudy Maskov. Mat. Obšc. 31, English transl., Trans. Mascow Math. Sac. 31, 81-148.

M. A. Krasnosel'skii, Ya. B. Rutickii (1961), Convex functions and Orlicz spaces (P. Noordhoff Ltd., Groningen, The Netherlands).

M. Krżyzánski (1964), 'Sur les solutions non négatives de l'équation linéaire normale parabolique', Rev. Roumaine Math. Pures Appl. (5) 9, 393-408.

A. Kufner, O. John, S. Fučik (1977), Function spaces, (Noordhoff International Publishing, Leyden, Academia Publishing House of the Czechoslovak Academy of Science, Prague).

H. Milicer-Grużewska (1960), 'Recherches sur les propriétés de la solution d'un système parabolique d'équations', Mem. Accad. Sci. Torino Cl. Sci. Fis. Mat. Natur. (3) 4, 255-280.

H. Milicer-Gruzewska (1963), 'Quelques propriétés des solutions fondamentales du système parabolique d'équations', Publ. Sém. Géom. Univ. Neuchatel, (1) 3, 3-9.

C. A. Rogers (1970), Hausdorff measures (Cambridge University Press).

C. A. Rogers and S. J. Taylor (1961), 'Functions continuous and singular with respect to a Hausdorff measure', Mathematica 8, 1-31. 
E. M. Stein (1970), Singular integrals and differentiability properties of functions (Princeton University Press, Princeton, New Jersey).

J. Szarski (1975), 'Strong maximum principle for non-linear parabolic differential functional inequalities in arbitrary domains', Ann. Polon. Math. 31, 197-303.

N. A. Watson (1974), 'Uniqueness and representation theorems for parabolic equations', J. London Math. Soc. 8, 311-321.

N. A. Watson (1977), 'Differentiation of measures and initial values of temperatures', J. London Math. Soc. 16, 271-282.

N. A. Watson (1978), 'The rate of special decay of non-negative solutions of linear parabolic equations', Arch. Rational Mech. Anal. 68, 121-124.

N. A. Watson (1980), 'Initial singularities of Gauss-Weierstrass integrals and their relations to Laplace transform and Hausdorff measures', to appear.

D. A. Widder (1944), 'Positive temperature on an infinite rod', Trans. Amer. Math. Soc. 55, 85-95.

C. H. Wilcox (1980), 'Positive temperatures with prescribed initial heat distributions', Amer. Math. Monthly 87, 183-186.

Department of Mathematics

The University of Queensland

St. Lucia, Queensland 4067

Australia 